\title{
A NEYMAN-PEARSON PERSPECTIVE ON OPTIMAL REINSURANCE WITH CONSTRAINTS
}

BY

Ambrose Lo

\begin{abstract}
The formulation of optimal reinsurance policies that take various practical constraints into account is a problem commonly encountered by practitioners. In the context of a distortion-risk-measure-based optimal reinsurance model without moral hazard, this article introduces and employs a variation of the Neyman-Pearson Lemma in statistical hypothesis testing theory to solve a wide class of constrained optimal reinsurance problems analytically and expeditiously. Such a Neyman-Pearson approach identifies the unit-valued derivative of each ceded loss function as the test function of an appropriate hypothesis test and transforms the problem of designing optimal reinsurance contracts to one that resembles the search of optimal test functions achieved by the classical Neyman-Pearson Lemma. As an illustration of the versatility and superiority of the proposed Neyman-Pearson formulation, we provide complete and transparent solutions of several specific constrained optimal reinsurance problems, many of which were only partially solved in the literature by substantially more difficult means and under extraneous technical assumptions. Examples of such problems include the construction of the optimal reinsurance treaties in the presence of premium budget constraints, counterparty risk constraints and the optimal insurer-reinsurer symbiotic reinsurance treaty considered recently in Cai et al. (2016).
\end{abstract}

\section{KEYWORDS}

Distortion, 1-Lipschitz, Value-at-Risk, reciprocal reinsurance, signed measure.

\section{INTRODUCTION}

The judicious use of reinsurance as a practical vehicle for risk optimization and strategic business planning lies at the heart of the successful operation of an insurance corporation. For more than half a century, reinsurance has provided a 
flexible mechanism on which insurance companies can capitalize to strategically transfer a portion of their business to reinsurers, thereby lessening the volatility of their risk exposure and achieving various business planning and solvency objectives. Pioneered in Borch (1960) and Arrow (1963) in the context of variance minimization and expected utility maximization from the perspective of an insurer, the study of optimal reinsurance has been established as a time-honored research area, which has gathered further momentum in recent decades given the increasingly intense need for risk management highlighted by the unprecedented surge in the frequency and severity of catastrophes worldwide.

Among the myriad of optimal reinsurance models in the literature, this article centers on the class of risk-measure-based models, which have gained widespread popularity alongside the recent proliferation of risk measures such as Value-at-Risk (VaR) and Tail Value-at-Risk (TVaR) in the banking and insurance industries. Unlike their classical expected utility counterparts, these models involve an explicit usage of risk measures to quantify risk, dictate preferences and formulate constraints. Adopting VaR and TVaR as risk measures, Cai and Tan (2007) determined analytically the optimal retention of a stop-loss reinsurance under the expected value premium principle. Their studies were continued in Cai et al. (2008), where the optimal reinsurance arrangements within the class of non-decreasing convex ceded loss functions were deduced by minimizing the VaR and TVaR of the total retained loss of the insurer via some sophisticated approximation and convergence arguments. Cheung (2010) revisited Cai et al. (2008)'s problems, provided an alternative geometric approach and also solved the VaR minimization problem under Wang's premium principle. Further developments along similar lines can be found in Chi and Tan (2011) and Tan et al. (2011). Since then, VaR-based and TVaR-based reinsurance models continued to arouse substantial attention from researchers and practitioners, and were extended in two predominant directions:

1. More general risk measures: Mainly of theoretical interest, one direction of extension is concerned with the use of risk measures and premium principles more general than VaR, TVaR and the standard expected value premium principle. Along this line, a particularly promising risk-measure-based optimal reinsurance model is the distortion-risk-measure-based model, which entails the minimization of the distortion risk measure (DRM) of the total retained risk of an insurer together with the distortion reinsurance premium principle. This model was first studied in Cui et al. (2013) under some technical left-continuity assumptions and later examined more transparently and systematically in Cheung and Lo (2017) from a cost-benefit perspective and in Zhuang et al. (2016) using a marginal indemnification function approach. The merits of the DRM-based model are multi-fold. First, it is known that the perception of risk DRM reflects is more consistent with human behavior than what the traditional expected utility paradigm offers (see Belles-Sampera et al., 2016 for more information). Second, due to the translation invariance of DRM, risk minimization is synonymous with 
profit maximization. Last but not least, the class of DRM is a very general one that includes VaR, TVaR and many other well-known risk measures as special cases. Although a general model usually leads to a higher level of technical complications, it turns out it is in the high level of generality embodied by the DRM setting that the mathematical structure of the reinsurance model becomes distinctively clear and the solution of the optimal reinsurance problem unexpectedly easy.

2. More complex constraints: Another promising line of research is devoted to the imposition of increasingly complex constraints that reflect diverse practical considerations, the most common one being a reinsurance premium budget constraint. In the context of a DRM-based model, the optimal reinsurance problem under a premium budget constraint was first studied in Cui et al. (2013), further examined in Zheng and Cui (2014), and later revisited and simplified in Cheung and Lo (2017) and Zhuang et al. (2016). The presence of such a budget constraint necessitates the allocation of limited financial resources to achieve minimality of risk and, as noted in Cheung and Lo (2017) and Zhuang et al. (2016), considerably raises the technical complexity of the optimal reinsurance problem.

Another popular constraint pertains to the risk tolerance level of the insurer and/or the reinsurer. This kind of constraint recognizes the two-party nature of reinsurance, which suggests that a reinsurance treaty designed solely from the perspective of one party while completely disregarding the interests of the other is practically non-marketable. In this direction, Cummins and Mahul (2004) were among the first to revisit Arrow's classical expected-utility-based optimal reinsurance model via imposing a deterministic upper bound on each feasible ceded loss function. In the framework of risk-measure-based models, the investigation of optimal reinsurance taking reinsurer's risk constraints into account was initiated in Cheung et al. (2012), where the TVaR of the insurer's total retained loss was minimized under a VaR constraint on the reinsurer's net loss. Recently, Lu et al. (2016) examined the problem of minimizing the VaR and TVaR of the insurer's total loss subject to bounded reinsurer's loss and provided an implicit construction of the optimal solutions. Whereas most studies are from the insurer's perspective only, Cai et al. (2016) took into account the joint interests of the insurer and reinsurer, and introduced an innovative problem in which a convex combination of the insurer's loss and the reinsurer's loss is minimized subject to their respective risk constraints.

In brief, there can be many economically meaningful constraints that can be embedded in an optimal reinsurance model, yet ad hoc approaches have been developed to (partially) solve a very limited number of constrained optimal reinsurance problems.

Motivated by the generality and usefulness of the DRM-based model and the diversity of constraints that prevail in practice, this paper strives to interweave the two foregoing lines of research, one theoretical and one practical, 
and introduces how a novel "Neyman-Pearson" perspective provides a unifying, transparent and comprehensive treatment of a wide body of constrained optimal reinsurance problems. The linchpin is a constrained functional minimization problem of considerable generality whose objective and constraint functions are both Lebesgue integrals with generic integrands carrying the unitvalued derivative of the ceded loss function to be chosen. It is shown that a number of existing and novel constrained optimal reinsurance problems, such as optimal contract design in the presence of a reinsurance premium budget, a counterparty risk constraint and Cai et al. (2016)'s insurer-reinsurer symbiotic problem, fall under the umbrella of such a Neyman-Pearson formulation upon appropriate configuration. When viewed in the correct light, the generic problem parallels the Neyman-Pearson Lemma in statistical hypothesis testing theory and can be solved via analogous comparison arguments, although it has its own subtly unique and technical issues. It is this striking resemblance between the proposed constrained minimization problem and the Neyman-Pearson Lemma that justifies designating our analysis as a "Neyman-Pearson" approach.

Given the wide variety of methods proposed in the recent literature to tackle constrained optimal reinsurance problems, it is imperative to pinpoint the fundamental differences and the superiority of the proposed Neyman-Pearson methodology relative to existing approaches with respect to the following three inter-related areas:

1. Scope of applicability: Compared to the cost-benefit argument first implicitly used in Cui et al. (2013) and later made precise in Cheung and Lo (2017), the Neyman-Pearson approach can be applied to deal with constraints much more general than reinsurance premium constraints, including many that are not cost-benefit in nature. When the constrained minimization problem under consideration does not possess a cost-benefit character, which relies on the non-negativity of the integrand of the constraint function, the approaches of Cui et al. (2013) and Cheung and Lo (2017) become inapplicable, yet the Neyman-Pearson solution scheme can be implemented just as easily. In this regard, the Neyman-Pearson approach can be considered as a non-trivial and versatile extension of the cost-benefit approach to one for analyzing more general and complex constraints.

2. Level of technicalities: Technically, our Neyman-Pearson methodology successfully puts constrained optimal reinsurance problems into perspective and provides a much more accessible and methodical approach to dealing with these problems, many of which were only partially solved in the literature by substantially more cumbersome means, under unnecessary mathematical assumptions and with particular solutions having been found. Ad hoc methods that very much hinge upon specific risk measures such as VaR and TVaR and are often plagued by superfluous technicalities (e.g., those in Cheung et al., 2012; Lu et al., 2016) are eschewed.

3. Explicitness and completeness of solutions: Arguably, the greatest strength of the Neyman-Pearson approach is its ability to exhibit the optimal 
solutions of a wide spectrum of constrained optimal reinsurance problems in explicit and easily comprehensible forms, as opposed to the nebulous solutions often accompanied by several implicitly defined quantities observed in many existing papers (e.g., Cai et al., 2016; Lu et al., 2016). In addition, with explicit solutions beyond the classical insurance-layer-type solutions identified, our approach manages to provide full characterizations of the optimal solutions and thoroughly address the feasibility, well-posedness and uniqueness issues of optimal reinsurance problems, which are unsettled in most papers (e.g., Cai et al., 2016; Lo, 2016; Lu et al., 2016).

The rest of this article is organized as follows. Section 2 describes the DRMbased optimal reinsurance model that forms the cornerstone of the entire article and presents three concrete models whose diversity and commonality motivate the Neyman-Pearson formulation of constrained optimal reinsurance problems proposed in Section 3. Heuristics that underlies the Neyman-Pearson solution scheme is explained, followed by its successive applications in Section 4 to fully solve three types of constrained optimal reinsurance problems. The closed-form solutions obtained are compared and contrasted with existing ones, and their superiority is highlighted. Section 5 concludes the article with prospective research directions.

\section{DRM-BASED OPTIMAL REINSURANCE MODEL}

In this section, we lay the mathematical groundwork of the DRM-based optimal reinsurance model, and explain the motivation of a Neyman-Pearson formulation of constrained optimal reinsurance problems via three concrete examples.

\subsection{Model set-up}

We begin with a precise and concise description of the DRM-based model paying special attention to the stipulation of the optimization criterion, the class of feasible ceded loss functions and the reinsurance premium principle. In this model, risk is evaluated by means of DRMs, whose definition relies on the notion of a distortion function. We say that $g:[0,1] \rightarrow[0,1]$ is a distortion function if $g$ is a non-decreasing function such that $g(0)=0$ and $g(1)=1$. Unlike many previous papers (such as Cui et al., 2013; Zhuang et al., 2016), we do not confine ourselves to convex, concave or (left- or right-) continuous distortion functions, since our analysis is valid without these extraneous technical assumptions and therefore of higher generality. Corresponding to a distortion function $g$, the DRM of a non-negative random variable $Y$ is defined by

$$
\rho_{g}(Y):=\int_{0}^{\infty} g\left(S_{Y}(y)\right) \mathrm{d} y
$$


where $S_{Y}(y):=\mathbb{P}(Y>y)$ is the survival function of $Y$. In order that (2.1) makes sense, throughout this article, we restrict our attention to distortion functions $g$ such that $g\left(0_{+}\right):=\lim _{x \downarrow 0} g(x)=0,{ }^{1}$ and assume that all random variables are sufficiently integrable in the sense of possessing well-defined and finite DRMs.

A remarkable feature of DRM is its inclusion of a wide class of common risk measures. In the later part of this article, we specifically analyze VaR, which is arguably the most popular DRM. Its definition is recalled below. In the sequel, we denote the indicator function of a given set $A$ by $1_{A}$, i.e., $1_{A}(x)=1$ if $x \in A$, and $1_{A}(x)=0$ otherwise.

Definition 2.1. (Definitions of generalized inverses and VaR). Given a subset $D$ of the real line and a non-decreasing function $T: D \rightarrow \mathbb{R}$, the generalized left-continuous inverse and generalized right-continuous inverse of $T$ are defined, respectively, by

$$
T^{-1}(x):=\inf \{y \in D \mid T(y) \geq x\} \quad \text { and } \quad T^{-1+}(x):=\inf \{y \in D \mid T(y)>x\},
$$

with the convention $\inf \emptyset=\sup D$. The VaR of a random variable $Y$ at the probability level of $p \in(0,1]$ is the generalized left-continuous inverse of its distribution function $F_{Y}$ at $p$ :

$$
\operatorname{VaR}_{p}(Y):=F_{Y}^{-1}(p)=\inf \left\{y \in \mathbb{R} \mid F_{Y}(y) \geq p\right\} .
$$

In this article, both the notation $\operatorname{VaR}_{p}(Y)$ and $F_{Y}^{-1}(p)$ will be used interchangeably whenever convenient. For later purposes, we point out the useful equivalence

$$
F_{Y}^{-1}(p) \leq y \quad \Leftrightarrow \quad p \leq F_{Y}(y) \text { for all } p \in(0,1),
$$

and that the distortion function giving rise to the $p$-level VaR is $g(x)=1_{\{x>1-p\}}$ (see Equations (4) and (44) of Dhaene et al., 2006), which is neither convex nor concave.

To mitigate its risk exposure to the ground-up loss, which is modeled by a general non-negative random variable $X$ (note that no extra assumptions such as absolute continuity or strict monotonicity besides non-negativity are imposed on the distribution of $X$ ), the insurer is offered the choice of purchasing a reinsurance policy $I$ from a reinsurer. When $x$ is the realization of $X$, the reinsurer pays $I(x)$ to the insurer, with the insurer retaining a loss of $x-I(x)$. Technically, the function $I$ is known as the ceded loss function (also known as the indemnity function) and defines a reinsurance policy. In this article, the feasible class of reinsurance policies available for sale is restricted to the set of non-decreasing and 1-Lipschitz functions null at zero, ${ }^{2}$ i.e.,

$\mathcal{I}=\left\{I: \mathbb{R}^{+} \rightarrow \mathbb{R}^{+} \mid I(0)=0, \quad 0 \leq I\left(x_{2}\right)-I\left(x_{1}\right) \leq x_{2}-x_{1}\right.$ for $\left.0 \leq x_{1} \leq x_{2}\right\}$.

The conditions imposed on the set $\mathcal{I}$ are not driven by mathematical considerations, but are intended to alleviate the practical issue of ex post moral hazard due to the manipulation of losses. In fact, for any reinsurance treaty selected from 
$\mathcal{I}$, the ceded loss cannot exceed the ground-up loss, and both the insurer and reinsurer will suffer as the ground-up loss becomes more severe, thereby having no incentive to misrepresent claims. Mathematically, the 1-Lipschitz condition does result in some appealing technical convenience, with every $I \in \mathcal{I}$ being absolutely continuous with a unit-valued derivative $I^{\prime}$, which exists almost everywhere. Following Zhuang et al. (2016), we term $I^{\prime}$ the marginal ceded loss function, which quantifies the increase in the ceded loss per unit increase in the ground-up loss. Moreover, because of the relationship $I(x)=\int_{0}^{x} I^{\prime}(t) \mathrm{d} t$, each ceded loss function enjoys a one-to-one correspondence with its marginal counterpart.

In exchange for bearing the ceded loss $I(X)$ from the insurer, the reinsurer is compensated by the reinsurance premium $P_{I(X)}$, which is a function of $I(X)$. The resulting total risk exposure of the insurer is then changed from $X$ to $X-I(X)+P_{I(X)}$. Conceivably, ceding more loss to the reinsurer results in the insurer incurring a smaller retained loss at the expense of a higher reinsurance premium. In essence, optimal reinsurance is an art of how risk should be ceded and retained to achieve a certain sense of optimality, which, in the current article, is minimization with respect to a DRM. Premium-wise, we calibrate the reinsurance premium for a given ceded loss function $I \in \mathcal{I}$ by the formula

$$
P_{I(X)}:=\int_{0}^{\infty} r\left(S_{I(X)}(x)\right) \mathrm{d} x,
$$

where $r:[0,1] \rightarrow \mathbb{R}^{+}$is a non-decreasing function satisfying $r\left(0_{+}\right)=0$. It is important to point out that we do not require $r(1)=1$, thereby making allowances for profit loading by the reinsurer possible. For example, one may set $r(x)=(1+\theta) h(x)$ with $r(1)=1+\theta>1$, where $h$ is a distortion function and $\theta>0$ is the safety loading charged by the reinsurer. Premium principles incorporating different safety loadings on different layers of risk such as $r(x)=\left[\left(1+\theta^{1}\right) 1_{\{0 \leq x \leq c\}}+\left(1+\theta^{2}\right) 1_{\{c<x \leq 1\}}\right] h(x)$, where $0<\theta^{1}<\theta^{2}$ and $0<c<1$, are also feasible. Equation (2.3) is therefore flexible enough to accommodate a wide variety of safety loading structures (including a structure that charges no loading).

Just as DRMs are general enough to cover a wide class of risk measures, a number of common premium principles, including the well-known expected value premium principle, Wang's premium principle and maximum possible claims principles, can be retrieved from (2.3) by different specifications of the function $r$ (see Cui et al., 2013; Cheung and Lo, 2017). Furthermore, the form of (2.3) is dual to (2.1). When viewed in the correct light, such symmetry is highly conducive to understanding the general mathematical structure of our optimal reinsurance models and turns out to put the analysis into much better perspective than when specific risk measures and premium principles are assumed.

The following technical lemma, which can be found in Lemma 2.1 of Cheung and Lo (2017), places the marginal ceded loss function $I^{\prime}$ in the integrands of appropriate integrals and renders the optimal selection of $I^{\prime}$ (equivalently, $I$ ) more 
transparent. It plays an instrumental role in the Neyman-Pearson formulation of optimal reinsurance problems.

Lemma 2.2. (Integral representations of risk and premium functions). For any distortion function $g$ and ceded loss function $I$ in $\mathcal{I}$,

$$
\rho_{g}\left(X-I(X)+P_{I(X)}\right)=\rho_{g}(X)+\int_{0}^{\infty}\left[r\left(S_{X}(x)\right)-g\left(S_{X}(x)\right)\right] I^{\prime}(x) \mathrm{d} x
$$

and

$$
P_{I(X)}=\int_{0}^{\infty} r\left(S_{X}(x)\right) I^{\prime}(x) \mathrm{d} x
$$

\subsection{Three concrete models}

In the context of the DRM-based model set up in the preceding subsection, we now introduce three sets of concrete constrained optimal reinsurance problems whose diversity with respect to their objective and constraint functions warrants the unifying Neyman-Pearson approach to be presented in Section 3.

- Model 1: Budget-constrained risk minimization

$$
\begin{cases}\inf _{I \in \mathcal{I}} & \rho_{g_{i}}\left(X-I(X)+P_{I(X)}\right) \\ \text { s.t. } & P_{I(X)} \leq \pi,\end{cases}
$$

where $g_{i}$ is the DRM selected by the insurer, and $\pi$ is a constant which can be thought of as the budget that the insurer allocates to reinsurance activities. In this model, the presence of the budget constraint reflects the financial limitations of the insurer, which must determine how to make the most of the limited budget to attain the greatest reduction in its total retained risk measured by the DRM. Problem (2.4), in the DRM framework, has its genesis in Cui et al. (2013), where some sophisticated partition arguments were employed under some technical assumptions on the functions $g_{i}$ and $r$. It was subsequently solved in complete generality and more transparently in Cheung and Lo (2017), who showed that the design of optimal reinsurance amounts to comparing the marginal cost and benefit of reinsurance.

- Model 2: Risk minimization in the presence of counterparty risk constraints As noted in Cai et al. $(2013,2016)$, the insurer and reinsurer, who are counterparties of each other, often have conflicting interests. Devising a reinsurance contract from the sole perspective of one party at the expense of the interests of the other, though mathematically feasible, is practically unrealistic. Consequently, a more pragmatic approach to optimal reinsurance is to formulate reinsurance policies which are optimal to one party and simultaneously 
acceptable to the other, as in the following two problems:

$$
\begin{cases}\inf _{I \in \mathcal{I}} & \rho_{g_{i}}\left(X-I(X)+P_{I(X)}\right) \\ \text { s.t. } & \rho_{g_{r}}\left(I(X)-P_{I(X)}\right) \leq \pi_{0},\end{cases}
$$

and

$$
\begin{cases}\inf _{I \in \mathcal{I}} & \rho_{g_{r}}\left(I(X)-P_{I(X)}\right) \\ \text { s.t. } & \rho_{g_{i}}\left(X-I(X)+P_{I(X)}\right) \leq \pi_{1},\end{cases}
$$

where $g_{r}$ is the distortion function adopted by the reinsurer to quantify its net risk exposure $I(X)-P_{I(X)}$ as a result of entering into the reinsurance contract. Economically, Problem (2.5) (resp. (2.6)) concerns the reinsurance arrangement which is optimal to the insurer (resp. reinsurer) while acceptable to the reinsurer (resp. insurer), whose risk level is maintained below the risk tolerance level of $\pi_{0}$ (resp. $\pi_{1}$ ). The counterparty risk constraints in Problems (2.5) and (2.6) are sometimes known as participation constraints because they induce the counterparty to "participate" in reinsurance.

It should be emphasized that Problems (2.5) and (2.6), which are in the general realm of DRM, are entirely novel and not amenable to existing approaches. Despite their generality and apparent intractability, it will be shown that our Neyman-Pearson solution scheme lends itself perfectly to Problems (2.5) and (2.6) for any distortion functions $g_{i}$ and $g_{r}$. In order to make the effects of the counterparty risk constraints more conspicuous, we will also examine the following specific VaR-based problems :

$$
\begin{cases}\inf _{I \in \mathcal{I}} & \operatorname{VaR}_{\alpha}\left(X-I(X)+P_{I(X)}\right) \\ \text { s.t. } & \operatorname{VaR}_{\beta}\left(I(X)-P_{I(X)}\right) \leq \pi_{0},\end{cases}
$$

and

$$
\begin{cases}\inf _{I \in \mathcal{I}} & \operatorname{VaR}_{\beta}\left(I(X)-P_{I(X)}\right) \\ \text { s.t. } & \operatorname{VaR}_{\alpha}\left(X-I(X)+P_{I(X)}\right) \leq \pi_{1},\end{cases}
$$

where $\alpha$ and $\beta$ are fixed probability levels in $(0,1)$ chosen by the insurer and reinsurer, respectively. The use of VaR in these two problems is justified by the widespread popularity and mathematical tractability of VaR. Note that a special case of Problem (2.7) with $\beta=1$ (i.e., bounded reinsurer's loss) was solved partially in the recent paper Lu et al. (2016) via some implicit constructions. As mentioned in Section 1 and will be shown later, one of the highlights of the Neyman-Pearson approach in this article is its ability to exhaust the entire set of optimal solutions of Problems (2.7) and (2.8) (as well as the general Problems (2.5) and (2.6)) explicitly and expeditiously. 
- Model 3: An insurer-reinsurer symbiotic optimal reinsurance model

$$
\left\{\begin{aligned}
\inf _{I \in \mathcal{I}} & \lambda \rho_{g_{i}}\left(X-I(X)+P_{I(X)}\right) & +(1-\lambda) \rho_{g_{r}}\left(I(X)-P_{I(X)}\right) \\
\text { s.t. } & \rho_{g_{i}}\left(X-I(X)+P_{I(X)}\right) & \leq \pi_{1}, \\
& \rho_{g_{r}}\left(I(X)-P_{I(X)}\right) & \leq \pi_{0},
\end{aligned}\right.
$$

where $\lambda$ is a number in $[0,1]$ that can be interpreted as the weight allocated to the insurer's loss, and $\pi_{1}$ and $\pi_{0}$ are again the risk tolerance levels of the insurer and reinsurer, respectively. First studied in Cai et al. (2016) in the special case when the insurer and reinsurer are both VaR-adopters, this "symbiotic" problem (in which the insurer and reinsurer form a "symbiosis") is another attempt to devise optimal reinsurance policies taking into account the possibly conflicting interests of the insurer and the reinsurer. Instead of minimizing the loss of one party subject to the risk constraint of the other, Problem (2.9) distinguishes itself from Problems (2.5) and (2.6) in that it entails the minimization of a convex combination of the DRM of the insurer's loss and that of the reinsurer's loss, subject to the risk constraints of both parties. Here, the weight $\lambda$ quantifies the relative importance of the interests of the insurer and reinsurer, with the special cases $\lambda=1$ and $\lambda=0$ corresponding to Problem (2.5) and Problem (2.6), respectively. The practical significance of Problem (2.9) consists in the formulation of reinsurance contracts that are not a priori designed to be optimal to one single party, but are compromised to be optimal on a symbiotic basis. Our prime interest lies in how this compromise manifests mathematically in the contract design, and in particular, depends on the choice of the weight $\lambda$. Intuitively, as $\lambda$ increases from 0 to 1 , more and more importance is attached to the insurer's interest, and it is expected that the solutions of Problem (2.9) will approach those of Problem (2.7).

In this article, we revisit Cai et al. (2016)'s VaR problem as a mathematically tractable and practically popular representative of Problem (2.9):

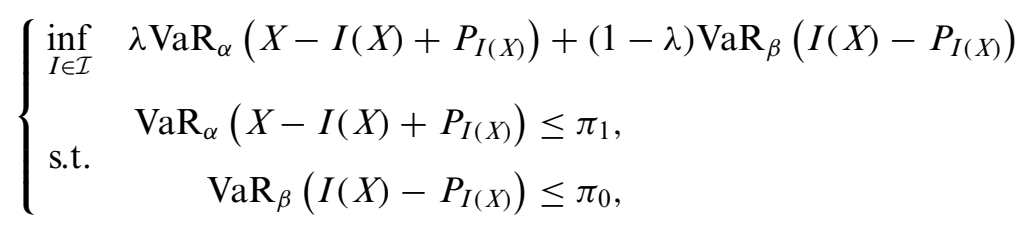

Through a series of sophisticated analysis involving the introduction of various auxiliary functions, Cai et al. (2016) derived the semi-explicit solutions of Problem (2.10). The approach pursued in this paper is radically different. The key is to exploit the properties of VaR as a member of the DRM and to transform Problem (2.10), in which two risk constraints are in force, equivalently into either Problem (2.7) or Problem (2.8), both of which carry a single risk constraint, depending on the range of values of $\lambda$. Such a transformation is unanticipated because it effectively means that the symbiotic 
risk-minimization problem is identical to the seemingly different risk minimization problem from the perspective of one party subject to the constraint of the other, when VaR is chosen as the measurement of risk. As soon as this first-step transformation has been performed, Problem (2.10) can be explicitly solved by virtue of the explicit solutions of Problems (2.7) and (2.8). In addition to being considerably more transparent, the solution scheme in this article enjoys the advantage of being able to characterize the entire set of solutions of Problem (2.10).

The aforementioned three sets of constrained optimal reinsurance problems possess different objective functions and/or constraint functions, as well as underlying economic meaning. Although developing model-specific, ad hoc solutions that are of limited applicability is possible, as is traditionally carried out in the literature, the conceptually and practically important question arises naturally as to whether it is feasible to develop a unifying framework to analyze all of these problems. Doing so not only places a large class of constrained optimal reinsurance problems under the same umbrella, but also puts the mathematical structure of these problems into perspective. The next section provides an affirmative answer, but one is prompted to view these constrained problems from a much more general and wider perspective, making their commonalities self-evident.

\section{NEYMAN-PEARSON FOR MULATION OF CONSTRAINED OPTIMAL REINSURANCE PROBLEMS}

The key to a unifying treatment of the constrained optimal reinsurance problems in the preceding section consists in recasting their objective and constraint functions in integral form aided by the use of Lemma 2.2, yielding

$$
\left\{\begin{array}{ll}
\inf _{I \in \mathcal{I}} & \int_{0}^{\infty} f_{1}(x) I^{\prime}(x) \mathrm{d} x \\
\text { s.t. } & \int_{0}^{\infty} f_{0}(x) I^{\prime}(x) \mathrm{d} x \leq \pi
\end{array},\right.
$$

where $f_{1}$ and $f_{0}$ are integrable functions defined on the non-negative real line, and $\pi$ is a generic real constant. Problem (3.1) is an abstract constrained functional minimization problem which not only is of theoretical interest per se, but also encompasses various practical constrained optimal reinsurance problems, including but not limited to those described in Section 2.2, ${ }^{3}$ upon the prescription of appropriate $f_{1}$ and $f_{0}$. It merits mention that in Problem (3.1), we do not assume the non-negativity or monotonicity of $f_{1}$ and $f_{0}$, neither is $\pi$ required to be a non-negative constant. This contributes to the generality of Problem (3.1) and, concomitantly, gives rise to some non-trivial technical challenges that need to be overcome meticulously. 
To statisticians, Problem (3.1) is strongly reminiscent of the classical result of the Neyman-Pearson Lemma in statistical hypothesis test theory (see, for example, Section 6.1 of Shao, 2003 and Section 3.2 of Lehmann and Romano, 2005). Before setting forth the formal solutions of Problem (3.1), it is instructive to unravel and exploit its intricate connections with the Neyman-Pearson Lemma. In fact, it will be shown later that Problem (3.1) can be solved by a modification of the essential spirit that underlies the Neyman-Pearson Lemma. To this end, we consider the maximization counterpart of Problem (3.1):

$$
\left\{\begin{array}{ll}
\sup _{I \in \mathcal{I}} & \int_{0}^{\infty} f_{1}(x) I^{\prime}(x) \mathrm{d} x \\
\text { s.t. } & \int_{0}^{\infty} f_{0}(x) I^{\prime}(x) \mathrm{d} x \leq \pi
\end{array} .\right.
$$

If $f_{1}$ and $f_{0}$ are non-negative integrable functions, then they can be thought of as kernels (i.e., unnormalized probability density functions) of two probability density functions, or equivalently as the Radon-Nikodým derivatives of two measures with respect to the Lebesgue measure on the non-negative real line. Introducing the two simple hypotheses

$$
\mathrm{H}_{0} \text { : The kernel of } X \text { is } f_{0} \text { versus } \quad \mathrm{H}_{1} \text { : The kernel of } X \text { is } f_{1}
$$

for any absolutely continuous ${ }^{4}$ non-negative random variable $X$ and identifying $I^{\prime}$ as the test function of a (randomized) hypothesis test (i.e., the probability of rejecting the null hypothesis) based on a single realization of $X$, one observes that Problem (3.2) can be phrased in probabilistic language as $\left(\mathbb{E}_{\mathrm{H}_{i}}\right.$ is the expectation under $\mathrm{H}_{i}$ for $i=0,1$ )

$$
\left\{\begin{array}{cl}
\sup _{I \in \mathcal{I}} & \mathbb{E}_{\mathrm{H}_{1}}\left[I^{\prime}(X)\right] \\
\text { s.t. } & \mathbb{E}_{\mathrm{H}_{0}}\left[I^{\prime}(X)\right] \leq \alpha \in(0,1)
\end{array},\right.
$$

which is equivalent to maximizing the power of the hypothesis test subject to the constraint that the size of the test is no larger than $\alpha$. The Neyman-Pearson Lemma asserts that the most powerful test is achieved by the likelihood ratio test (LRT), which rejects the null hypothesis for certain if the likelihood ratio $f_{1}(X) / f_{0}(X)$ takes sufficiently large values, i.e., the optimal test function is

$$
I_{*}^{\prime}(x)=1_{\left\{f_{1}(x)>c^{*} f_{0}(x)\right\}}+\gamma_{*}(x) 1_{\left\{f_{1}(x)=c^{*} f_{0}(x)\right\}},
$$

for some positive cutoff level $c^{*}$ and unit-valued function $\gamma_{*}$ so that the test has an exact size of $\alpha$. This comparison spirit makes intuitive sense because the null hypothesis is justifiably rejected when the alternative hypothesis appears considerably more plausible as supported by a large alternative likelihood $f_{1}$, in conjunction with an untenable null hypothesis suggested by a low null likelihood $f_{0}$. 
Upon comparing Problem (3.1) with the classical Neyman-Pearson problem, the most conspicuous difference (apart from the fact that minimization instead of maximization is performed in the former) is that $f_{1}$ and $f_{0}$ in Problem (3.1) are not necessarily non-negative functions, as is the case in most constrained optimal reinsurance problems. This precludes the interpretation of $f_{1}$ and $f_{0}$ as kernels of two absolutely continuous probability distributions and deprives Problem (3.1) of the colorful probabilistic meaning in connection with the Neyman-Pearson Lemma. Despite this noteworthy technical difference, we shall see in Theorem 3.2 that essentially the same comparison spirit underlying the Neyman-Pearson Lemma is at work and the optimal solutions of Problem (3.1) are still of the LRT type, provided that appropriate modifications taking into account the signs of $f_{1}$ and $f_{0}$ are made (see Remark 3.3 (i)).

We begin with a preparatory lemma concerned with some useful technical properties of the constraint function when restricted to the class of LRT-type reinsurance policies. Below, we denote by $G\left(c_{+}\right)$and $G\left(c_{-}\right)$the right-hand and left-hand limits of $G$ at any point $c$.

Lemma 3.1. (Technical properties of the restricted constraint function). Define $G:[-\infty, 0] \rightarrow \mathbb{R}$ by

$$
G(c):=\int_{\left\{f_{1}<c f_{0}\right\}} f_{0}(x) \mathrm{d} x .
$$

Then,

a. $G$ is a non-decreasing function.

b. $G(0)=\int_{\left\{f_{1}<0\right\}} f_{0}(x) \mathrm{d} x$ and $G(-\infty)=\int_{\left\{f_{0}<0\right\}} f_{0}(x) \mathrm{d} x$.

c. For any $c<0$,

$$
G(c)-G\left(c_{-}\right)=-\int_{\left\{f_{1}=c f_{0}, f_{0}<0\right\}} f_{0}(x) \mathrm{d} x
$$

and

$$
G\left(c_{+}\right)-G(c)=\int_{\left\{f_{1}=c f_{0}, f_{0}>0\right\}} f_{0}(x) \mathrm{d} x .
$$

Proof. a. The fact that $G$ is non-decreasing can be easily seen by writing

$$
G(c)=\int_{\left\{f_{1} / f_{0}<c, f_{0}>0\right\}} f_{0}(x) \mathrm{d} x+\int_{\left\{f_{1} / f_{0}>c, f_{0}<0\right\}} f_{0}(x) \mathrm{d} x,
$$

where both integrals are non-decreasing in $c$.

b. These follow immediately from the definition of $G$ and (3.3).

c. These follow from the fact that the first integral in (3.3) is left-continuous in $c$, whereas the second integral is right-continuous in $c$.

In the classical Neyman-Pearson setting, $f_{1}$ and $f_{0}$ are non-negative, in which case $G$ is a non-decreasing left-continuous function. In the general 
context of Problem (3.1), $f_{1}$ and $f_{0}$ are signed integrable functions, resulting in the technical complication that $G$, although non-decreasing, is not necessarily a left-continuous or right-continuous function. See the proof of Proposition 4.3 for an example where $G$ exhibits both left-discontinuity and right-discontinuity.

Equipped with Lemma 3.1, we are now in a position to fully solve Problem (3.1). Our proof parallels that of the classical Neyman-Pearson Lemma, but additional technical difficulties introduced by the signed nature of $f_{1}$ and $f_{0}$ need to be tackled. For simplicity, the solutions of Problem (3.1) are equivalently and more conveniently stated in terms of the marginal ceded loss function. No generality is lost due to the one-to-one correspondence between a ceded loss function and its marginal counterpart.

Theorem 3.2. (Solutions of generalized Neyman-Pearson Problem (3.1)). Consider Problem (3.1).

a. Suppose that $\pi \geq G(0)=\int_{\left\{f_{1}<0\right\}} f_{0}(x) \mathrm{d} x$. Then, the optimal solutions of Problem (3.1) must be in the form of

$$
I_{*}^{\prime}(x)= \begin{cases}1, & \text { if } f_{1}(x)<0 \\ \gamma_{*}(x), & \text { if } f_{1}(x)=0 \\ 0, & \text { if } f_{1}(x)>0\end{cases}
$$

where $\gamma_{*}: \mathbb{R}^{+} \rightarrow[0,1]$ is any function such that

$$
\int_{0}^{\infty} f_{0}(x) I_{*}^{\prime}(x) \mathrm{d} x=G(0)+\int_{\left\{f_{1}=0\right\}} f_{0}(x) \gamma_{*}(x) \mathrm{d} x \leq \pi .
$$

b. Suppose that

$$
\int_{\left\{f_{0}<0\right\}} f_{0}(x) \mathrm{d} x=G(-\infty) \leq \pi<G(0)=\int_{\left\{f_{1}<0\right\}} f_{0}(x) \mathrm{d} x .
$$

Define $c^{*}=G^{-1+}(\pi)$. Then, the optimal solutions of Problem (3.1) must be of the form

$$
I_{*}^{\prime}(x)= \begin{cases}1, & \text { if } f_{1}(x)<c^{*} f_{0}(x), \\ \gamma_{*}(x), & \text { if } f_{1}(x)=c^{*} f_{0}(x), \\ 0, & \text { if } f_{1}(x)>c^{*} f_{0}(x),\end{cases}
$$

where $\gamma_{*}: \mathbb{R}^{+} \rightarrow[0,1]$ is any function such that

$$
\int_{0}^{\infty} f_{0}(x) I_{*}^{\prime}(x) \mathrm{d} x=G\left(c^{*}\right)+\int_{\left\{f_{1}=c^{*} f_{0}\right\}} f_{0}(x) \gamma_{*}(x) \mathrm{d} x=\pi .
$$

c. If $\pi<G(-\infty)=\int_{\left\{f_{0}<0\right\}} f_{0}(x) \mathrm{d} x$, then Problem (3.1) has no solutions. 
Proof. Since Case (c) is trivial and the proofs of Cases (a) and (b) are analogous to each other, to avoid unnecessary repetition, we only prove (b), which is the most interesting and intricate case.

We first show that the selection of a unit-valued function $\gamma_{*}: \mathbb{R}^{+} \rightarrow[0,1]$ such that

$$
\int_{0}^{\infty} f_{0}(x) I_{*}^{\prime}(x) \mathrm{d} x=G\left(c^{*}\right)+\int_{\left\{f_{1}=c^{*} f_{0}\right\}} f_{0}(x) \gamma_{*}(x) \mathrm{d} x=\pi
$$

is always possible. By the definition of $c^{*}$, three cases can arise:

Case (1). Assume that $G\left(c_{-}^{*}\right) \leq \pi<G\left(c^{*}\right)$. By Lemma 3.1 (c), we have $\int_{\left\{f_{1}=c^{*} f_{0}, f_{0}<0\right\}} f_{0}(x) \mathrm{d} x<0$, and

$$
\begin{aligned}
G\left(c_{-}^{*}\right)=G\left(c^{*}\right)+\int_{\left\{f_{1}=c^{*} f_{0}, f_{0}<0\right\}} & f_{0}(x) \mathrm{d} x \\
& \leq G\left(c^{*}\right)+\int_{\left\{f_{1}=c^{*} f_{0}, f_{0}<0\right\}} f_{0}(x) \gamma(x) \mathrm{d} x \leq G\left(c^{*}\right)
\end{aligned}
$$

for any unit-valued function $\gamma$. To satisfy (3.4), a possible choice of $\gamma_{*}(\cdot)$ is to set

$$
\gamma_{*}(x)=\frac{\pi-G\left(c^{*}\right)}{\int_{\left\{f_{1}=c^{*} f_{0}, f_{0}<0\right\}} f_{0}(t) \mathrm{d} t} 1_{\left\{f_{0}(x)<0\right\}} \in[0,1] \text { for all } x \geq 0 .
$$

Case (2). Assume that $G\left(c^{*}\right)=\pi$. Then, setting $\gamma_{*}(x)=0$ for all $x \geq 0$ immediately leads to (3.4).

Case (3). Assume that $G\left(c^{*}\right)<\pi \leq G\left(c_{+}^{*}\right)$. By Lemma 3.1 (c) again, we have $\int_{\left\{f_{1}=c^{*} f_{0}, f_{0}>0\right\}} f_{0}(x) \mathrm{d} x>0$, and

$$
\begin{aligned}
G\left(c^{*}\right) \leq G\left(c^{*}\right)+\int_{\left\{f_{1}=c^{*} f_{0}, f_{0}>0\right\}} & f_{0}(x) \gamma(x) \mathrm{d} x \\
\leq & G\left(c^{*}\right)+\int_{\left\{f_{1}=c^{*} f_{0}, f_{0}>0\right\}} f_{0}(x) \mathrm{d} x=G\left(c_{+}^{*}\right)
\end{aligned}
$$

for any unit-valued function $\gamma$. We can set

$$
\gamma_{*}(x)=\frac{\pi-G\left(c^{*}\right)}{\int_{\left\{f_{1}=c^{*} f_{0}, f_{0}>0\right\}} f_{0}(t) \mathrm{d} t} 1_{\left\{f_{0}(x)>0\right\}} \in[0,1] \text { for all } x \geq 0
$$

to fulfill (3.4).

We now fix any $I \in \mathcal{I}$ such that $\int_{0}^{\infty} f_{0}(x) I^{\prime}(x) \mathrm{d} x \leq \pi$. If $f_{1}(x)<c^{*} f_{0}(x)$, then $I_{*}^{\prime}(x)-I^{\prime}(x)=1-I^{\prime}(x) \geq 0$. When $f_{1}(x)>c^{*} f_{0}(x)$, we have 
$I_{*}^{\prime}(x)-I^{\prime}(x)=-I^{\prime}(x) \leq 0$. Therefore,

$$
\left[f_{1}(x)-c^{*} f_{0}(x)\right]\left[I_{*}^{\prime}(x)-I^{\prime}(x)\right] \leq 0 \text { for any } x \geq 0,
$$

so integrating both sides over the non-negative real line yields

$$
\int_{0}^{\infty}\left[f_{1}(x)-c^{*} f_{0}(x)\right]\left[I_{*}^{\prime}(x)-I^{\prime}(x)\right] \mathrm{d} x \leq 0,
$$

which is equivalent to

$$
\int_{0}^{\infty} f_{1}(x)\left[I_{*}^{\prime}(x)-I^{\prime}(x)\right] \mathrm{d} x \leq c^{*} \int_{0}^{\infty} f_{0}(x)\left[I_{*}^{\prime}(x)-I^{\prime}(x)\right] \mathrm{d} x .
$$

Because $c^{*} \leq 0$ and $\int_{0}^{\infty} f_{0}(x) I^{\prime}(x) \mathrm{d} x \leq \pi=\int_{0}^{\infty} f_{0}(x) I_{*}^{\prime}(x) \mathrm{d} x$, we conclude that

$$
\int_{0}^{\infty} f_{1}(x)\left[I_{*}^{\prime}(x)-I^{\prime}(x)\right] \mathrm{d} x \leq 0,
$$

establishing the minimality of $I_{*}$.

If $I_{* *}$ is another function in $\mathcal{I}$ such that $\int_{0}^{\infty} f_{1}(x) I_{* *}^{\prime}(x) \mathrm{d} x=$ $\int_{0}^{\infty} f_{1}(x) I_{*}^{\prime}(x) \mathrm{d} x$ and $\int_{0}^{\infty} f_{0}(x) I_{* *}^{\prime}(x) \mathrm{d} x \leq \pi$, then necessarily

$$
\int_{0}^{\infty}\left[f_{1}(x)-c^{*} f_{0}(x)\right]\left[I_{*}^{\prime}(x)-I_{* *}^{\prime}(x)\right] \mathrm{d} x=0 .
$$

This, together with $\left[f_{1}(x)-c^{*} f_{0}(x)\right]\left[I_{*}^{\prime}(x)-I_{* *}^{\prime}(x)\right] \leq 0$ for all $x \geq 0$, implies that $I_{* *}^{\prime}=I_{*}^{\prime}$ almost everywhere on $\left\{f_{1} \neq c^{*} f_{0}\right\}$.

\section{Remark 3.3.}

i. The construction of the optimal solutions in Case (b) of Theorem 3.2 can be demystified by the dissection analysis performed in Lo (2016), which can be summarized as follows. Observe that the set $\left\{f_{1}<c^{*} f_{0}\right\}$ can be partitioned into

$$
\begin{aligned}
\left\{f_{1}<c^{*} f_{0}\right\}= & \left\{f_{1}<c^{*} f_{0}, f_{0} \leq 0\right\} \cup\left\{f_{1}<c^{*} f_{0}, f_{0}>0\right\} \\
= & \left\{f_{1}<0, f_{0} \leq 0\right\} \cup\left\{c^{*}<f_{1} / f_{0}<0, f_{0}<0\right\} \\
& \cup\left\{f_{1} / f_{0}<c^{*}, f_{0}>0\right\},
\end{aligned}
$$

in which the three sets reflect different heuristic considerations:

- Every optimal solution $I_{*}$ of Problem (3.1) must satisfy $I_{*}^{\prime}(x)=1$ for all $x \in\left\{f_{1}<0, f_{0} \leq 0\right\}$, on which the objective and constraint functions are simultaneously reduced to the greatest possible extent. Likewise, we must have $I_{*}^{\prime}(x)=0$ for all $x \in\left\{f_{1}>0, f_{0} \geq 0\right\}$.

- On the set $\left\{f_{1}<0, f_{0}>0\right\}$, an extra unit of ceded loss lowers the objective function but undesirably raises the constraint function. To result in the greatest decrease in the objective function with the least increase 
in the constraint function, intuitively one should set $I_{*}^{\prime}(x)=1$ on the subset where $f_{1}$ takes the most negative values while $f_{0}$ assumes the least positive values, or equivalently, where the ratio $f_{1} / f_{0}$ takes the most negative values. This translates into the subset $\left\{f_{1} / f_{0}<c^{*}, f_{0}>0\right\}=\left\{f_{1}<\right.$ $\left.c^{*} f_{0}, f_{0}>0\right\}$.

- On the set $\left\{f_{1}>0, f_{0}<0\right\}$, optimally we set $I_{*}^{\prime}(x)=1$ on the subset, where $f_{1}$ takes the least positive values, whereas $f_{0}$ assumes the most negative values, or where $f_{1} / f_{0}$ takes the least negative values. This is captured by the subset $\left\{c^{*}<f_{1} / f_{0}<0, f_{0}<0\right\}$.

More details of such dissection analysis can be found in Subsection 3.1 of Lo (2016).

ii. A careful examination of the proof of Theorem 3.2 reveals that $c^{*}$ can be set to any value between (and including) $G^{-1}(\pi)$ and $G^{-1+}(\pi)$. Regardless of the choice of $c^{*}$, the three mutually exclusive and exhaustive cases (1) $G\left(c_{-}^{*}\right) \leq \pi<G\left(c^{*}\right)$; (2) $G\left(c^{*}\right)=\pi$ and (3) $G\left(c^{*}\right)<\pi \leq G\left(c_{+}^{*}\right)$ should still be distinguished because the existence of $\gamma_{*}$ needs to be handled separately.

iii. If the set of ceded loss functions is changed to

$$
\mathcal{I}=\left\{I: \mathbb{R}^{+} \rightarrow \mathbb{R}^{+} \mid I(0)=0, \quad I_{1}^{\prime}(x) \leq I^{\prime}(x) \leq I_{2}^{\prime}(x) \text { for all } x \geq 0\right\},
$$

where $I_{1}^{\prime}$ and $I_{2}^{\prime}$ are two given unit-valued functions, then the optimal solutions of Problem (3.1) can be easily modified as

$$
I_{*}^{\prime}(x)= \begin{cases}I_{2}^{\prime}(x), & \text { if } f_{1}(x)<c^{*} f_{0}(x), \\ \gamma_{*}(x), & \text { if } f_{1}(x)=c^{*} f_{0}(x), \\ I_{1}^{\prime}(x), & \text { if } f_{1}(x)>c^{*} f_{0}(x),\end{cases}
$$

where $\gamma_{*}: \mathbb{R}^{+} \rightarrow[0,1]$ is any unit-valued function such that $I_{1}^{\prime}(x) \leq \gamma_{*}(x) \leq$ $I_{2}^{\prime}(x)$ for all $x \geq 0$, and $\int_{0}^{\infty} f_{0}(x) I_{*}^{\prime}(x) \mathrm{d} x \leq \pi$ in Case (a) of Theorem 3.2 and $\int_{0}^{\infty} f_{0}(x) I_{*}^{\prime}(x) \mathrm{d} x=\pi$ in Case (b), with other obvious modifications on the definition of the restricted constraint function $G$ and the three conditions on $\pi$ in Theorem 3.2. Such constraints on the class of feasible ceded loss functions were imposed in Balbás et al. (2015) and Zhuang et al. (2016) to further reduce moral hazard.

iv. In essence, Theorem 3.2 is a generalization of the classical Neyman-Pearson Lemma from (probability) measures to signed measures defined by the two possibly signed functions $f_{1}$ and $f_{0}$, and $I^{\prime}$ admits more general interpretations than a test function (in this article, $I^{\prime}$ is interpreted as a marginal ceded loss function, but it need not be in other contexts). Note that while there exist scattered extensions of the Neyman-Pearson Lemma to signed measures akin to Theorem 3.2 in the literature, these results are often stated in an incomplete and obscure form that is not useful for practical purposes. One example is Theorem 1 of Wagner (1969), in which the class of feasible ceded loss functions (following the terminology and notation of the current article) is defined in terms of the optimal solution given a priori, 
not to mention that additional differentiability conditions are imposed on $f_{1}$ and $f_{0}$. Another example is Proposition 10 on page 30 of Rao (2014), where the optimality of $I_{*}$ is established only among all $I \in \mathcal{I}$ such that $\int_{0}^{\infty} f_{0}(x) I^{\prime}(x) \mathrm{d} x=\int_{0}^{\infty} f_{0}(x) I_{*}^{\prime}(x) \mathrm{d} x$, but not all $I \in \mathcal{I}$ satisfying the constraint $\int_{0}^{\infty} f_{0}(x) I^{\prime}(x) \mathrm{d} x \leq \pi$, and that the value of $\pi$ is not clearly distinguished. With Theorem 3.2, we provide a clear-cut characterization of the entire set of optimal solutions of Problem (3.1) for all mutually exclusive and exhaustive conditions on $\pi$. In doing so, it also addresses the issue of (non)uniqueness of the optimal solutions of Problem (3.1). It is shown that the solutions are generally non-unique, unless the set $\left\{f_{1}=c^{*} f_{0}\right\}$ is a Lebesgue null set.

\section{ClOSED-FORM SOLUTIONS OF THREE CONCRETE CONSTRAINED OPTIMAL REINSURANCE PROBLEMS}

Theorem 3.2 forms the theoretical foundation of our Neyman-Pearson approach to optimal reinsurance and furnishes a ready recipe for solving a wide body of constrained optimal reinsurance problems efficiently. To illustrate the versatility of Theorem 3.2, in this section we prescribe the functions $f_{1}$ and $f_{0}$ in Problem (3.1), and employ Theorem 3.2 to completely solve the three concrete constrained optimal reinsurance problems presented in Subsection 2.2.

\subsection{Budget-constrained optimal reinsurance}

The budget-constrained optimal reinsurance problem studied in a number of prior papers can be solved effortlessly as an immediate application of Theorem 3.2 .

Proposition 4.1. (Budget-constrained optimal reinsurance). Consider Problem (2.4).

a. Suppose that $\pi \geq \int_{\left\{r\left(S_{X}\right)<g_{i}\left(S_{X}\right)\right\}} r\left(S_{X}(x)\right) \mathrm{d} x$. Then, Problem (2.4) is solved by

$$
I_{*}^{\prime}(x)= \begin{cases}1, & \text { if } r\left(S_{X}(x)\right)<g_{i}\left(S_{X}(x)\right), \\ \gamma_{*}(x), & \text { if } r\left(S_{X}(x)\right)=g_{i}\left(S_{X}(x)\right), \\ 0, & \text { if } r\left(S_{X}(x)\right)>g_{i}\left(S_{X}(x)\right),\end{cases}
$$

for any function $\gamma_{*}: \mathbb{R}^{+} \rightarrow[0,1]$ such that

$$
\int_{\left\{r\left(S_{X}\right)<g_{i}\left(S_{X}\right)\right\}} r\left(S_{X}(x)\right) \mathrm{d} x+\int_{\left\{r\left(S_{X}\right)=g_{i}\left(S_{X}\right)\right\}} r\left(S_{X}(x)\right) \gamma_{*}(x) \mathrm{d} x \leq \pi .
$$


b. Suppose that $0 \leq \pi<\int_{\left\{r\left(S_{X}\right)<g_{i}\left(S_{X}\right)\right\}} r\left(S_{X}(x)\right) \mathrm{d} x$. Then, Problem (2.4) is solved by

$$
I_{*}^{\prime}(x)= \begin{cases}1, & \text { if } g_{i}\left(S_{X}(x)\right) / r\left(S_{X}(x)\right)>c^{*}, \\ \gamma_{*}(x), & \text { if } g_{i}\left(S_{X}(x)\right) / r\left(S_{X}(x)\right)=c^{*}, \\ 0, & \text { if } g_{i}\left(S_{X}(x)\right) / r\left(S_{X}(x)\right)<c^{*},\end{cases}
$$

where $c^{*}=\inf \left\{c \geq 1 \mid \int_{\left\{g_{i}\left(S_{X}\right) / r\left(S_{X}\right)>c\right\}} r\left(S_{X}(x)\right) \mathrm{d} x \leq \pi\right\}$, and $\gamma_{*}: \mathbb{R}^{+} \rightarrow$ $[0,1]$ is any function such that

$$
\int_{\left\{g_{i}\left(S_{X}\right) / r\left(S_{X}\right)>c^{*}\right\}} r\left(S_{X}(x)\right) \mathrm{d} x+\int_{\left\{g_{i}\left(S_{X}\right) / r\left(S_{X}\right)=c^{*}\right\}} r\left(S_{X}(x)\right) \gamma_{*}(x) \mathrm{d} x=\pi .
$$

c. Suppose that $\pi<0$. Then, Problem (2.4) has no solutions.

Proof. In this setting, $f_{1}(x)=r\left(S_{X}(x)\right)-g_{i}\left(S_{X}(x)\right)$ and $f_{0}(x)=r\left(S_{X}(x)\right)$. Then, Cases (a) and (c) are immediate. For Case (b), it suffices to note that

$$
\begin{aligned}
c^{*} & =\sup \left\{c \leq 0 \mid \int_{\left\{r\left(S_{X}\right)-g_{i}\left(S_{X}\right)<c r\left(S_{X}\right)\right\}} r\left(S_{X}(x)\right) \mathrm{d} x \leq \pi\right\} \\
& =\sup \left\{c \leq 0 \mid \int_{\left\{g_{i}\left(S_{X}\right) / r\left(S_{X}\right)>1-c\right\}} r\left(S_{X}(x)\right) \mathrm{d} x \leq \pi\right\} \\
& =1-\inf \left\{c \geq 1 \mid \int_{\left\{g_{i}\left(S_{X}\right) / r\left(S_{X}\right)>c\right\}} r\left(S_{X}(x)\right) \mathrm{d} x \leq \pi\right\},
\end{aligned}
$$

followed by relabeling $1-c^{*}$ as $c^{*}$.

Proposition 4.1 is a streamlined version of Theorem 3.3 of Cheung and Lo (2017) and Theorem 4.1 of Zhuang et al. (2016). It shows that their cost-benefit and marginal indemnification function approaches fall into the realm of the Neyman-Pearson approach of this article. As amply discussed in Cheung and Lo (2017), $g_{i}\left(S_{X}\right) / r\left(S_{X}\right)$ can be interpreted as the ratio of the marginal benefit of reinsurance to the marginal cost of reinsurance. Intuition suggests and Proposition 4.1 (b) confirms that the insurer, with the aim of minimizing its total retained risk using the given limited budget, should purchase reinsurance on the losses on which the excess of the marginal benefit of reinsurance over the marginal cost is the greatest, until the whole budget is consumed. We refer to Cheung and Lo (2017) for more details.

\subsection{Optimal reinsurance in the presence of counterparty risk constraints}

We now analyze the insurer's DRM minimization problem subject to the reinsurer's DRM risk constraint (Problem (2.5)) and the reinsurer's DRM minimization problem subject to the insurer's DRM risk constraint (Problem (2.6)). Due to the close resemblance between the two problems, we only present the 
solutions for Problem (2.5), for which the functions $f_{1}$ and $f_{0}$ are given by

$$
f_{1}(x)=r\left(S_{X}(x)\right)-g_{i}\left(S_{X}(x)\right) \quad \text { and } \quad f_{0}(x)=g_{r}\left(S_{X}(x)\right)-r\left(S_{X}(x)\right) .
$$

Specializing Theorem 3.2 to these two functions immediately leads to the following complete solutions of Problem (2.5).

Proposition 4.2. (Insurer's DRM minimization in the presence of reinsurer's DRM risk constraint). Consider Problem (2.5). Define $G:[-\infty, 0] \rightarrow \mathbb{R}$ by

$$
G(c)=\int_{\left\{(1+c) r\left(S_{X}\right)<g_{i}\left(S_{X}\right)+c g_{r}\left(S_{X}\right)\right\}}\left[g_{r}\left(S_{X}(x)\right)-r\left(S_{X}(x)\right)\right] \mathrm{d} x .
$$

a. Suppose that $\pi \geq \int_{\left\{r\left(S_{X}\right)<g_{i}\left(S_{X)}\right\}\right.}\left[g_{r}\left(S_{X}(x)\right)-r\left(S_{X}(x)\right)\right] \mathrm{d} x$. Then, Problem (2.5) is solved by

$$
I_{*}^{\prime}(x)= \begin{cases}1, & \text { if } r\left(S_{X}(x)\right)<g_{i}\left(S_{X}(x)\right), \\ \gamma_{*}(x), & \text { if } r\left(S_{X}(x)\right)=g_{i}\left(S_{X}(x)\right), \\ 0, & \text { if } r\left(S_{X}(x)\right)>g_{i}\left(S_{X}(x)\right),\end{cases}
$$

for any function $\gamma_{*}: \mathbb{R}^{+} \rightarrow[0,1]$ such that

$$
G(0)+\int_{\left\{r\left(S_{X}\right)=g_{i}\left(S_{X}\right)\right\}}\left[g_{r}\left(S_{X}(x)\right)-r\left(S_{X}(x)\right)\right] \gamma_{*}(x) \mathrm{d} x \leq \pi .
$$

b. Suppose that

$$
\begin{aligned}
& \int_{\left\{g_{r}\left(S_{X}\right)<r\left(S_{X}\right)\right\}}\left[g_{r}\left(S_{X}(x)\right)-r\left(S_{X}(x)\right)\right] \mathrm{d} x \leq \pi \\
& \quad<\int_{\left\{r\left(S_{X}\right)<g_{i}\left(S_{X}\right)\right\}}\left[g_{r}\left(S_{X}(x)\right)-r\left(S_{X}(x)\right)\right] \mathrm{d} x .
\end{aligned}
$$

Define $c^{*}=G^{-1+}(\pi)$. Then, Problem (2.5) is solved by

$$
I_{*}^{\prime}(x)= \begin{cases}1, & \text { if }\left(1+c^{*}\right) r\left(S_{X}(x)\right)<g_{i}\left(S_{X}(x)\right)+c^{*} g_{r}\left(S_{X}(x)\right), \\ \gamma_{*}(x), & \text { if }\left(1+c^{*}\right) r\left(S_{X}(x)\right)=g_{i}\left(S_{X}(x)\right)+c^{*} g_{r}\left(S_{X}(x)\right), \\ 0, & \text { if }\left(1+c^{*}\right) r\left(S_{X}(x)\right)>g_{i}\left(S_{X}(x)\right)+c^{*} g_{r}\left(S_{X}(x)\right),\end{cases}
$$

where $\gamma_{*}: \mathbb{R}^{+} \rightarrow[0,1]$ is any function such that

$$
G\left(c^{*}\right)+\int_{\left\{\left(1+c^{*}\right) r\left(S_{X}\right)=g_{i}\left(S_{X}\right)+c^{*} g_{r}\left(S_{X}\right)\right\}}\left[g_{r}\left(S_{X}(x)\right)-r\left(S_{X}(x)\right)\right] \gamma_{*}(x) \mathrm{d} x=\pi .
$$

c. Suppose that $\pi<\int_{\left\{g_{r}\left(S_{X}\right)<r\left(S_{X}\right)\right\}}\left[g_{r}\left(S_{X}(x)\right)-r\left(S_{X}(x)\right)\right] \mathrm{d} x$. Then, Problem (2.5) has no solutions. 
We remark that our solutions given in Proposition 4.2 work for any distortion functions $g_{i}$ and $g_{r}$ because our methodology based on Theorem 3.2 does not rely on any particular properties of the functions $f_{1}$ and $f_{0}$.

In order to display the above optimal solutions more concretely and to cast further light on the effects of the counterparty risk constraints on the contract design, in the remainder of this subsection, we concentrate on the VaR-based Problems (2.7) and (2.8) as exemplars of Problems (2.5) and (2.6) with particular practical relevance (owing to the ubiquity of VaR) and with partial solutions available in $\mathrm{Lu}$ et al. (2016), whereby the utility of the Neyman-Pearson approach fully manifests itself. For concreteness, we suppose that the expected value premium principle with a profit loading of $\theta$ is in force, i.e., $r(x)=(1+\theta) x$, where $\theta \geq 0$. Extensively used in the literature, including Lu et al. (2016), this reinsurance premium principle facilitates the comparison of our novel results with those in $\mathrm{Lu}$ et al. (2016), although our methodology remains valid when other premium principles in the general form of (2.3) are prescribed (at the possible cost of losing the concrete representations of the optimal ceded loss functions). The functions $f_{1}$ and $f_{0}$ are then given by

$$
f_{1}(x)=(1+\theta) S_{X}(x)-1_{\left\{S_{X}(x)>1-\alpha\right\}} \text { and } f_{0}(x)=1_{\left\{S_{X}(x)>1-\beta\right\}}-(1+\theta) S_{X}(x) .
$$

In what follows, we write, for any real $x$ and $y, x \wedge y=\min (x, y), x \vee y=$ $\max (x, y)$ and $x_{+}=\max (x, 0)$.

Proposition 4.3. (Insurer's VaR minimization in the presence of reinsurer's VaR risk constraint). Consider Problem (2.7) with $f_{1}$ and $f_{0}$ specified in (4.1). Assume that $\theta /(\theta+1) \leq \alpha \wedge \beta$.

a. Suppose that $\pi_{0} \geq \int_{F_{X}^{-1+}(\theta /(\theta+1))}^{F_{-1}^{-1}(x)} f_{0}(x) \mathrm{d} x$. Then, Problem (2.7) is solved by

$$
I_{*}^{\prime}(x)= \begin{cases}1, & \text { if } F_{X}^{-1+}(\theta /(\theta+1))<x<F_{X}^{-1}(\alpha), \\ \gamma_{*}(x), & \text { if } F_{X}^{-1}(\theta /(\theta+1)) \leq x \leq F_{X}^{-1+}(\theta /(\theta+1)), \\ 0, & \text { elsewhere }\end{cases}
$$

for any function $\gamma_{*}: \mathbb{R}^{+} \rightarrow[0,1]$ such that

$$
\int_{F_{X}^{-1+}(\theta /(\theta+1))}^{F_{X}^{-1}(\alpha)} f_{0}(x) \mathrm{d} x+\int_{F_{X}^{-1}(\theta /(\theta+1))}^{F_{X}^{-1+}(\theta /(\theta+1))} f_{0}(x) \gamma_{*}(x) \mathrm{d} x \leq \pi_{0} .
$$

b. Suppose that

$$
\int_{\left[0, F_{X}^{-1}(\theta /(\theta+1))\right) \cup\left[F_{X}^{-1}(\beta), \infty\right)} f_{0}(x) \mathrm{d} x \leq \pi_{0}<\int_{F_{X}^{-1+}(\theta /(\theta+1))}^{F_{X}^{-1}(\alpha)} f_{0}(x) \mathrm{d} x .
$$


Then, Problem (2.7) is solved by

$$
I_{*}^{\prime}(x)= \begin{cases}1, & \text { if } F_{X}^{-1}(\beta) \leq x<F_{X}^{-1}(\alpha), \\ \gamma_{*}(x), & \text { if } 0 \leq x<F_{X}^{-1}(\alpha \wedge \beta) \text { or } x \geq F_{X}^{-1}(\alpha \vee \beta), \\ 0, & \text { elsewhere, }\end{cases}
$$

for any function $\gamma_{*}: \mathbb{R}^{+} \rightarrow[0,1]$ such that

$$
\int_{\left[F_{X}^{-1}(\beta), F_{X}^{-1}(\alpha)\right)} f_{0}(x) \mathrm{d} x+\int_{\left[0, F_{X}^{-1}(\alpha \wedge \beta)\right) \cup\left[F_{X}^{-1}(\alpha \vee \beta), \infty\right)} f_{0}(x) \gamma_{*}(x) \mathrm{d} x=\pi_{0} .
$$

c. Suppose that $\pi_{0}<\int_{\left[0, F_{X}^{-1}(\theta /(\theta+1))\right) \cup\left[F_{X}^{-1}(\beta), \infty\right)} f_{0}(x) \mathrm{d} x$. Then, Problem (2.7) admits no solutions.

Proof. For any fixed $c \leq 0$, consider the inequality $(1+c) r\left(S_{X}(x)\right)<$ $g_{i}\left(S_{X}(x)\right)+c g_{r}\left(S_{X}(x)\right)$, which is equivalent to

$$
(1+c)(1+\theta) S_{X}(x)<1_{\left\{S_{X}(x)>1-\alpha\right\}}+c 1_{\left\{S_{X}(x)>1-\beta\right\}}
$$

over $x \in\left[0, F_{X}^{-1}(1)\right)$. We distinguish three cases.

Case (1). When $c<-1$, then with the aid of (2.2), we see that (4.2) is true if and only if $x<F_{X}^{-1}(\theta /(\theta+1))$ or $x \geq F_{X}^{-1}(\beta)$.

Case (2). When $c=-1$, then (4.2) is valid if and only if $x \in\left[F_{X}^{-1}(\beta), F_{X}^{-1}(\alpha)\right)$ (which is an empty set when $\alpha \leq \beta$ ).

Case (3). When $c>-1$, then (4.2) holds if and only if either $x \in$ $\left(F_{X}^{-1+}(\theta /(\theta+1)), F_{X}^{-1}(\alpha)\right)$ or $x \in\left[F_{X}^{-1+}(\theta /(\theta+1)), F_{X}^{-1}(\alpha)\right)$ (the latter occurs when $F_{X}$ has a point of discontinuity at $\left.F_{X}^{-1+}(\theta /(\theta+1))\right)$.

Noting that almost everywhere equal functions have the same Lebesgue integral, we deduce that (see Figure 1)

$$
G(c)= \begin{cases}\int_{\left[0, F_{X}^{-1}(\theta /(\theta+1))\right) \cup\left[F_{X}^{-1}(\beta), \infty\right)} f_{0}(x) \mathrm{d} x, & \text { if } c<-1, \\ \int_{\left[F_{X}^{-1}(\beta), F_{X}^{-1}(\alpha)\right)} f_{0}(x) \mathrm{d} x, & \text { if } c=-1, \\ \int_{F_{X}^{-1+}(\theta /(\theta+1))}^{F_{X}^{-1}(\alpha)} f_{0}(x) \mathrm{d} x, & \text { if }-1<c \leq 0 .\end{cases}
$$

If $\pi_{0}$ satisfies

$$
\int_{\left[0, F_{X}^{-1}(\theta /(\theta+1))\right) \cup\left[F_{X}^{-1}(\beta), \infty\right)} f_{0}(x) \mathrm{d} x \leq \pi_{0}<\int_{F_{X}^{-1+}(\theta /(\theta+1))}^{F_{X}^{-1}(\alpha)} f_{0}(x) \mathrm{d} x
$$




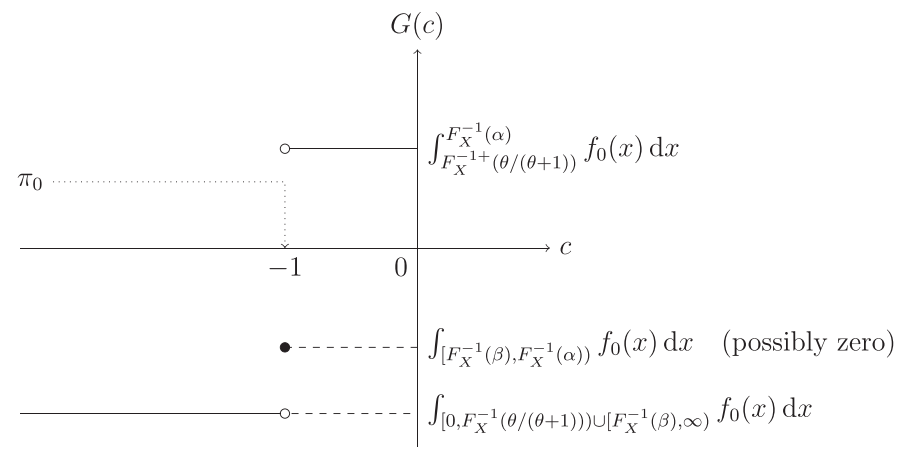

FIGURE 1: The graph of $G(c)$ in Proposition 4.3.

$$
\begin{aligned}
& \text { then } c^{*}=-1 \text {. As } \\
& \begin{aligned}
\left\{r\left(S_{X}\right)<g_{i}\left(S_{X}\right)\right\}= & \left(F_{X}^{-1+}(\theta /(\theta+1)), F_{X}^{-1}(\alpha)\right) \text { or }\left[F_{X}^{-1+}(\theta /(\theta+1)), F_{X}^{-1}(\alpha)\right), \\
\left\{r\left(S_{X}\right)=g_{i}\left(S_{X}\right)\right\}= & {\left[F_{X}^{-1}(\theta /(\theta+1)), F_{X}^{-1+}(\theta /(\theta+1))\right) \text { or } } \\
& {\left[F_{X}^{-1}(\theta /(\theta+1)), F_{X}^{-1+}(\theta /(\theta+1))\right], } \\
\left\{g_{r}\left(S_{X}\right)<r\left(S_{X}\right)\right\}= & {\left[F_{X}^{-1}(\beta), F_{X}^{-1}(\alpha)\right), } \\
\left\{g_{r}\left(S_{X}\right)=r\left(S_{X}\right)\right\}= & {\left[0, F_{X}^{-1}(\alpha \wedge \beta)\right) \cup\left[F_{X}^{-1}(\alpha \vee \beta), \infty\right), }
\end{aligned}
\end{aligned}
$$

an application of Proposition 4.2 completes the proof.

\section{Remark 4.4.}

i. The assumption $\theta /(\theta+1) \leq \alpha \wedge \beta$, which is imposed to ease the presentation of the optimal solutions, is indeed very mild and, for all intents and purposes, satisfied, because the profit loading $\theta$ charged by the reinsurer usually takes a small positive value whereas the probability levels $\alpha$ and $\beta$ that define the VaR risk measure tend to approach one in practice.

ii. Further insights into the behavior of the optimal solutions of Problem (2.7) can be gained by examining the signs and magnitudes of $f_{1}$ and $f_{0}$ over different subsets of the non-negative real line.

- Note that $f_{1}$ and $f_{0}$ take the same sign on the set $\left[F_{X}^{-1}(\alpha \wedge \beta), F_{X}^{-1}(\alpha \vee\right.$ $\beta)$ ). When $\alpha>\beta$, both $f_{1}$ and $f_{0}$ are negative on $\left[F_{X}^{-1}(\alpha \wedge \beta), F_{X}^{-1}(\alpha \vee\right.$ $\beta))=\left[F_{X}^{-1}(\beta), F_{X}^{-1}(\alpha)\right)$. The optimal reinsurance strategy is to purchase full coverage on this set (i.e., set $I_{*}^{\prime}(x)=1$ ), leading to the greatest reduction in the objective function, which aligns with the goal of Problem (2.7), as well as the constraint function, providing additional room for reinsurance coverage on other sets. When $\alpha \leq \beta$, both $f_{1}$ and $f_{0}$ are positive on $\left[F_{X}^{-1}(\alpha \wedge \beta), F_{X}^{-1}(\alpha \vee \beta)\right)=\left[F_{X}^{-1}(\alpha), F_{X}^{-1}(\beta)\right)$. Reinsurance coverage in this set thanklessly increases the objective function as well as 
the constraint function. This set should be completely discarded in the design of the optimal reinsurance treaty.

- Outside $\left[F_{X}^{-1}(\alpha \wedge \beta), F_{X}^{-1}(\alpha \vee \beta)\right), f_{1}$ and $f_{0}$ assume opposite signs but the same magnitude. Ceding an extra unit of loss on the complement of $\left[F_{X}^{-1}(\alpha \wedge \beta), F_{X}^{-1}(\alpha \vee \beta)\right)$ leads to a decrease (resp. increase) in the objective function that is exactly compensated by the increase (resp. decrease) in the constraint function. This explains the perhaps astounding result in Proposition 4.3 (b) that the design of the optimal reinsurance policy can be arbitrary on the complement of $\left[F_{X}^{-1}(\alpha \wedge \beta), F_{X}^{-1}(\alpha \vee \beta)\right)$, as long as the resulting contract binds the reinsurer's risk constraint.

iii. Proposition 4.3 can be considered as a substantial improvement of Theorem 3.6 of $\mathrm{Lu}$ et al. (2016) involving bounded reinsurer's risk in three respects:

1. First, Proposition 4.3 distinguishes various optimal solutions of Problem (2.7) by different explicit, mutually exclusive and exhaustive conditions on the upper bound $\pi_{0}$, which can be readily ascertained upon the prescription of the distribution of the ground-up loss $X$, in contrast to the conditions which are defined implicitly in Lu et al. (2016) (see the conditions " $b_{0}^{*} \geq \operatorname{VaR}_{X}(p)$ ", " $b_{0}^{*}<\operatorname{VaR}_{X}(p)$ " and the implicit definition of the quantity $b_{0}^{*}$ therein) and difficult to verify. Our conditions on $\pi_{0}$ are also mathematical ways of expressing the stringency of the reinsurer's risk constraint.

2. Second, with respect to the form of the optimal solutions, arguably the greatest power of Proposition 4.3 is that it manages to exhaust all of the solutions of Problem (2.7), whereas only particular insurancelayer-type solutions are presented in Theorem 3.6 of Lu et al. (2016). This is inherently due to Lu et al. (2016)'s methodology, which reduces the feasible set of ceded loss functions to a two-dimensional sub-class and inevitably results in a loss of optimal solutions. The NeymanPearson approach in this article is a clear demonstration that a wide spectrum of reinsurance policies can serve as the optimal solutions of Problem (2.7), in addition to providing an explicit and transparent characterization of the set of optimal solutions.

3. Third, Proposition 4.3 possesses much higher generality in that we dispense with the absolute continuity and strict monotonicity traditionally imposed on the distribution function of the ground-up loss for technical reasons, and that Problem (2.7) is defined by a VaR risk constraint characterized by the reinsurer's risk level $\beta$, which is generally different from the insurer's risk level $\alpha$. Proposition 4.3 not only encompasses the two complementary cases, $\alpha \leq \beta$ (in which case the set $\left[F_{X}^{-1}(\beta), F_{X}^{-1}(\alpha)\right)$ is empty and the integral $\int_{\left[F_{X}^{-1}(\beta), F_{X}^{-1}(\alpha)\right)} f_{0}(x) \mathrm{d} x$ is zero) and $\alpha>\beta$, but also retrieves the bounded loss setting in $\mathrm{Lu}$ et al. (2016) upon sending $\beta$ to 1 . 
With different specifications of $f_{1}$ and $f_{0}$, our Neyman-Pearson solution scheme can be readily adapted to solve the TVaR-based problems also studied in $\mathrm{Lu}$ et al. (2016).

As $f_{1}<c f_{0}$ is equivalent to $f_{0}<c^{-1} f_{1}$ for any $c<0$, a by-product of the proof of Proposition 4.3 is the following corollary, which provides complete solutions of Problem (2.8) and will be of use in the next subsection.

Corollary 4.5. (Reinsurer's VaR minimization in the presence of insurer's VaR risk constraint). Consider Problem (2.8) with $f_{1}$ and $f_{0}$ specified in (4.1). Assume that $\theta /(\theta+1) \leq \alpha \wedge \beta$.

a. Suppose that $\pi_{1} \geq \int_{\left[0, F_{X}^{-1}(\theta /(\theta+1))\right) \cup\left[F_{X}^{-1}(\beta), \infty\right)} f_{1}(x) \mathrm{d} x$. Then, Problem (2.8) is solved by

$$
I_{*}^{\prime}(x)= \begin{cases}1, & \text { if } x<F_{X}^{-1}(\theta /(\theta+1)) \text { or } x \geq F_{X}^{-1}(\beta), \\ \gamma_{*}(x), & \text { if } F_{X}^{-1}(\theta /(\theta+1)) \leq x \leq F_{X}^{-1}(\theta /(\theta+1)), \\ 0, & \text { if } F_{X}^{-1+}(\theta /(\theta+1))<x<F_{X}^{-1}(\beta),\end{cases}
$$

for any function $\gamma_{*}: \mathbb{R}^{+} \rightarrow[0,1]$ such that

$$
\int_{\left[0, F_{X}^{-1+}(\theta /(\theta+1))\right) \cup\left[F_{X}^{-1}(\beta), \infty\right)} f_{1}(x) \mathrm{d} x+\int_{F_{X}^{-1}(\theta /(\theta+1))}^{F_{X}^{-1+}(\theta /(\theta+1))} f_{1}(x) \gamma_{*}(x) \mathrm{d} x \leq \pi_{1} .
$$

b. Suppose that

$$
\int_{F_{X}^{-1+}(\theta /(\theta+1))}^{F_{X}^{-1}(\alpha)} f_{1}(x) \mathrm{d} x \leq \pi_{1}<\int_{\left[0, F_{X}^{-1}(\theta /(\theta+1))\right) \cup\left[F_{X}^{-1}(\beta), \infty\right)} f_{1}(x) \mathrm{d} x .
$$

Then, Problem (2.8) is solved by

$$
I_{*}^{\prime}(x)= \begin{cases}1, & \text { if } F_{X}^{-1}(\beta) \leq x<F_{X}^{-1}(\alpha), \\ \gamma_{*}(x), & \text { if } 0 \leq x<F_{X}^{-1}(\alpha \wedge \beta) \text { or } x \geq F_{X}^{-1}(\alpha \vee \beta), \\ 0, & \text { elsewhere, }\end{cases}
$$

for any function $\gamma_{*}: \mathbb{R}^{+} \rightarrow[0,1]$ such that

$$
\int_{\left[F_{X}^{-1}(\beta), F_{X}^{-1}(\alpha)\right)} f_{1}(x) \mathrm{d} x+\int_{\left[0, F_{X}^{-1}(\alpha \wedge \beta)\right) \cup\left[F_{X}^{-1}(\alpha \vee \beta), \infty\right)} f_{1}(x) \gamma_{*}(x) \mathrm{d} x=\pi_{1} .
$$

c. Suppose that $\pi_{1}<\int_{F_{X}^{-1+}(\theta /(\theta+1))}^{F_{-1}^{-1}(\alpha)} f_{1}(x) \mathrm{d} x$. Then, Problem (2.8) has no solutions. 


\subsection{Insurer-reinsurer symbiotic optimal reinsurance}

As a further illustration of the Neyman-Pearson technique and a natural continuation of Problems (2.7) and (2.8) studied in the preceding subsection, we now revisit the insurer-reinsurer symbiotic risk minimization problem (Problem (2.10)) studied in Cai et al. (2016), which we reproduce as follows for convenience:

$$
\left\{\begin{array}{cc}
\inf _{I \in \mathcal{I}} & \lambda \operatorname{VaR}_{\alpha}\left(X-I(X)+P_{I(X)}\right)+(1-\lambda) \operatorname{VaR}_{\beta}\left(I(X)-P_{I(X)}\right) \\
\text { s.t. } & \operatorname{VaR}_{\alpha}\left(X-I(X)+P_{I(X)}\right) \leq \pi_{1} \\
& \operatorname{VaR}_{\beta}\left(I(X)-P_{I(X)}\right) \leq \pi_{0}
\end{array} .\right.
$$

To guarantee that the set of solutions of Problem (2.10) is non-empty, we follow Cai et al. (2016) and impose the following assumptions on the ranges of values of $\pi_{1}$ and $\pi_{0}$ :

$$
\left\{\begin{array}{rl}
F_{X}^{-1+}\left(\frac{\theta}{\theta+1}\right)+(1+\theta) \int_{F_{X}^{-1+}(\theta /(\theta+1))}^{F_{X}^{-1}(\alpha)} S_{X}(x) \mathrm{d} x & \leq \pi_{1} \\
F_{X}^{-1}\left(\frac{\theta}{\theta+1}\right)-(1+\theta)\left(\int_{\left[0, F_{X}^{-1}(\theta /(\theta+1))\right) \cup\left[F_{X}^{-1}(\beta), \infty\right)} S_{X}(x) \mathrm{d} x\right) & \leq \pi_{0} \\
F_{X}^{-1}(\alpha \wedge \beta) & \leq \pi_{1}+\pi_{0}
\end{array} .\right.
$$

The first two conditions ensure that $\pi_{1}$ and $\pi_{0}$ are no less than the smallest values of the two constraint functions, i.e.,

$$
\inf _{I \in \mathcal{I}} \operatorname{VaR}_{\alpha}\left(X-I(X)+P_{I(X)}\right) \leq \pi_{1} \text { and } \inf _{I \in \mathcal{I}} \operatorname{VaR}_{\beta}\left(I(X)-P_{I(X)}\right) \leq \pi_{0}
$$

and the last condition, which arises from the 1-Lipschitz property of any $I \in \mathcal{I}$, rules out the possibility that $\pi_{1}$ and $\pi_{0}$ simultaneously take exceptionally low values. The allowable set of values of $\left(\pi_{1}, \pi_{0}\right)$ is then a triangular region, in which optimal solutions of Problem (2.10) exist, as will be shown in Proposition 4.6 below. Most intriguingly, Problem (2.10) can be solved by disregarding one of the two constraints and reducing it to either Problem (2.7) or Problem (2.8), depending on whether $\lambda>1 / 2$ or $\lambda<1 / 2$ (see Remark 4.7 (i) for why this happens). By doing so, the optimal solutions presented in Proposition 4.3 and Corollary 4.5 of Section 4.2 will come in useful again, and Problem (2.10) can be solved very easily.

Proposition 4.6. (Insurer-reinsurer symbiotic optimal reinsurance). Consider Problem (2.10) with $f_{1}$ and $f_{0}$ specified in (4.1). Assume that $\theta /(\theta+1) \leq \alpha \wedge \beta$. 
a. If $\lambda>1 / 2$, then Problem (2.10) has the same set of solutions as Problem (2.7), i.e.,

$$
\left\{\begin{array}{ll}
\inf _{I \in \mathcal{I}} & \operatorname{VaR}_{\alpha}\left(X-I(X)+P_{I(X)}\right) \\
\text { s.t. } & \operatorname{VaR}_{\beta}\left(I(X)-P_{I(X)}\right) \leq \pi_{0}
\end{array},\right.
$$

with $\pi_{0}$ satisfying (4.3) (see Proposition 4.3 for the solutions).

b. If $\lambda=1 / 2$, then Problem (2.10) is solved by

$$
I_{*}^{\prime}(x)= \begin{cases}1, & \text { if } F_{X}^{-1}(\beta) \leq x<F_{X}^{-1}(\alpha), \\ \gamma_{*}(x), & \text { if } 0 \leq x<F_{X}^{-1}(\alpha \wedge \beta) \text { or } x \geq F_{X}^{-1}(\alpha \vee \beta), \\ 0, & \text { elsewhere, }\end{cases}
$$

where $\gamma_{*}: \mathbb{R}^{+} \rightarrow[0,1]$ is any function such that

$$
\int_{\left[F_{X}^{-1}(\beta), F_{X}^{-1}(\alpha)\right)} f_{i}(x) \mathrm{d} x+\int_{\left[0, F_{X}^{-1}(\alpha \wedge \beta)\right) \cup\left[F_{X}^{-1}(\alpha \vee \beta), \infty\right)} f_{i}(x) \gamma_{*}(x) \mathrm{d} x \leq \pi_{i}
$$

for $i=0,1$.

c. If $\lambda<1 / 2$, then Problem (2.10) has the same set of solutions as Problem (2.8), i.e.,

$$
\left\{\begin{array}{ll}
\inf _{I \in \mathcal{I}} & \operatorname{VaR}_{\beta}\left(I(X)-P_{I(X)}\right) \\
\text { s.t. } & \operatorname{VaR}_{\alpha}\left(X-I(X)+P_{I(X)}\right) \leq \pi_{1}
\end{array},\right.
$$

with $\pi_{1}$ satisfying (4.3) (see Corollary 4.5 for the solutions).

Proof. We only prove (a) here. The proofs of (b) and (c) are similar and omitted. In integral form, Problem (2.10) is equivalent to

$$
\begin{cases}\inf _{I \in \mathcal{I}} & \int_{0}^{\infty}\left[\lambda f_{1}(x)+(1-\lambda) f_{0}(x)\right] I^{\prime}(x) \mathrm{d} x \\ & \int_{0}^{\infty} f_{1}(x) I^{\prime}(x) \mathrm{d} x \leq \pi_{1}^{\prime} \\ \text { s.t. } & \int_{0}^{\infty} f_{0}(x) I^{\prime}(x) \mathrm{d} x \leq \pi_{0}\end{cases}
$$

where $\pi_{1}^{\prime}=\pi_{1}-\operatorname{VaR}_{\alpha}(X)$. As $f_{1}$ and $f_{0}$ are both negative on $\left[F_{X}^{-1}(\beta), F_{X}^{-1}(\alpha)\right)$ and positive on $\left[F_{X}^{-1}(\alpha), F_{X}^{-1}(\beta)\right)$ (see Remark 4.4 (ii)), without loss of generality we may restrict attention to only those $I^{\prime}$ s such that $I^{\prime}(x)=1$ for all $x \in\left[F_{X}^{-1}(\beta), F_{X}^{-1}(\alpha)\right)$ and $I^{\prime}(x)=0$ for all $x \in\left[F_{X}^{-1}(\alpha), F_{X}^{-1}(\beta)\right)$, since every optimal solution (if it exists) of Problem (4.4) must satisfy these properties.

Suppose that $\lambda>1 / 2$, and let $I_{*}$ be any optimal solution of Problem (2.7) with $\pi_{0}$ satisfying (4.3). We first show that $I_{*}$ is a feasible solution of Problem 
(4.4). By Proposition 4.3, the optimal value of the objective function of Problem (2.7) is

$$
\begin{aligned}
\int_{0}^{\infty} f_{1}(x) I_{*}^{\prime}(x) \mathrm{d} x & =\int_{F_{X}^{-1+}(\theta /(\theta+1))}^{F_{X}^{-1}(\alpha)} f_{1}(x) \mathrm{d} x+\int_{F_{X}^{-1}(\theta /(\theta+1))}^{F_{X}^{-1+}(\theta /(\theta+1))} f_{1}(x) \gamma^{*}(x) \mathrm{d} x \\
& =F_{X}^{-1+}\left(\frac{\theta}{\theta+1}\right)+(1+\theta) \int_{F_{X}^{-1+}(\theta /(\theta+1))}^{F_{X}^{-1}(\alpha)} S_{X}(x) \mathrm{d} x-F_{X}^{-1}(\alpha) \\
& \leq \pi_{1}-F_{X}^{-1}(\alpha) \\
& =\pi_{1}^{\prime},
\end{aligned}
$$

if $\pi_{0} \geq \int_{F_{X}^{-1+}(\theta /(\theta+1))}^{F^{-1}(\alpha)} f_{0}(x) \mathrm{d} x$, by the first inequality in (4.3); if

$$
\int_{\left[0, F_{X}^{-1}(\theta /(\theta+1))\right) \cup\left[F_{X}^{-1}(\beta), \infty\right)} f_{0}(x) \mathrm{d} x \leq \pi_{0}<\int_{F_{X}^{-1+}(\theta /(\theta+1))}^{F_{X}^{-1}(\alpha)} f_{0}(x) \mathrm{d} x,
$$

then,

$$
\begin{aligned}
& \int_{0}^{\infty} f_{1}(x) I_{*}^{\prime}(x) \mathrm{d} x \\
= & \int_{\left[F_{X}^{-1}(\beta), F_{X}^{-1}(\alpha)\right)} f_{1}(x) \mathrm{d} x+\int_{\left[0, F_{X}^{-1}(\alpha \wedge \beta)\right) \cup\left[F_{X}^{-1}(\alpha \vee \beta), \infty\right)} f_{1}(x) \gamma^{*}(x) \mathrm{d} x \\
= & (1+\theta) \int_{\left[F_{X}^{-1}(\beta), F_{X}^{-1}(\alpha)\right)} S_{X}(x) \mathrm{d} x-\left[F_{X}^{-1}(\alpha)-F_{X}^{-1}(\beta)\right]_{+} \\
& \quad-\int_{\left[0, F_{X}^{-1}(\alpha \wedge \beta)\right) \cup\left[F_{X}^{-1}(\alpha \vee \beta), \infty\right)} f_{0}(x) \gamma^{*}(x) \mathrm{d} x \\
= & -\int_{\left[F_{X}^{-1}(\beta), F_{X}^{-1}(\alpha)\right)} f_{0}(x) \mathrm{d} x-\left[F_{X}^{-1}(\alpha)-F_{X}^{-1}(\beta)\right]_{+} \\
& -\int_{\left[0, F_{X}^{-1}(\alpha \wedge \beta)\right) \cup\left[F_{X}^{-1}(\alpha \vee \beta), \infty\right)} f_{0}(x) \gamma^{*}(x) \mathrm{d} x \\
= & -\pi_{0}-\left[F_{X}^{-1}(\alpha)-F_{X}^{-1}(\beta)\right]_{+} \\
\leq & \pi_{1}-F_{X}^{-1}(\alpha \wedge \beta)-\left[F_{X}^{-1}(\alpha)-F_{X}^{-1}(\beta)\right]_{+} \\
= & \pi_{1}-F_{X}^{-1}(\alpha) \\
= & \pi_{1}^{\prime},
\end{aligned}
$$

using the third inequality in (4.3). In both cases, $\int_{0}^{\infty} f_{1}(x) I_{*}^{\prime}(x) \mathrm{d} x \leq \pi_{1}^{\prime}$, showing the feasibility of $I_{*}$ as a solution of Problem (4.4). 
Now for any fixed $I$ satisfying $\int_{0}^{\infty} f_{1}(x) I^{\prime}(x) \mathrm{d} x \leq \pi_{1}^{\prime}, \int_{0}^{\infty} f_{0}(x) I^{\prime}(x) \mathrm{d} x \leq$ $\pi_{0}, I^{\prime}(x)=1$ for all $x \in\left[F_{X}^{-1}(\beta), F_{X}^{-1}(\alpha)\right)$ and $I^{\prime}(x)=0$ for all $x \in$ $\left[F_{X}^{-1}(\alpha), F_{X}^{-1}(\beta)\right)$, it follows from the definition of $I_{*}$ that

$$
\int_{0}^{\infty} f_{1}(x) I_{*}^{\prime}(x) \mathrm{d} x \leq \int_{0}^{\infty} f_{1}(x) I^{\prime}(x) \mathrm{d} x .
$$

Moreover, because $f_{1}(x)=-f_{0}(x)$ for all $x \notin\left[F_{X}^{-1}(\alpha \wedge \beta), F_{X}^{-1}(\alpha \vee \beta)\right)$ and $I_{*}^{\prime}(x)=I^{\prime}(x)$ for all $x \in\left[F_{X}^{-1}(\alpha \wedge \beta), F_{X}^{-1}(\alpha \vee \beta)\right)$, we also have

$$
\int_{0}^{\infty} f_{0}(x)\left[I^{\prime}(x)-I_{*}^{\prime}(x)\right] \mathrm{d} x=\int_{0}^{\infty} f_{1}(x)\left[I_{*}^{\prime}(x)-I^{\prime}(x)\right] \mathrm{d} x \leq 0 .
$$

Therefore,

$$
\begin{aligned}
& \int_{0}^{\infty}\left[\lambda f_{1}(x)+(1-\lambda) f_{0}(x)\right]\left[I_{*}^{\prime}(x)-I^{\prime}(x)\right] \mathrm{d} x \\
= & \lambda \int_{0}^{\infty} f_{1}(x)\left[I_{*}^{\prime}(x)-I^{\prime}(x)\right] \mathrm{d} x+(1-\lambda) \int_{0}^{\infty} f_{0}(x)\left[I_{*}^{\prime}(x)-I^{\prime}(x)\right] \mathrm{d} x \\
= & \lambda \int_{0}^{\infty} f_{1}(x)\left[I_{*}^{\prime}(x)-I^{\prime}(x)\right] \mathrm{d} x+(1-\lambda) \int_{0}^{\infty} f_{1}(x)\left[I^{\prime}(x)-I_{*}^{\prime}(x)\right] \mathrm{d} x \\
= & (2 \lambda-1) \int_{0}^{\infty} f_{1}(x)\left[I_{*}^{\prime}(x)-I^{\prime}(x)\right] \mathrm{d} x \\
\leq & 0
\end{aligned}
$$

where the last inequality follows from $\lambda>1 / 2$. This proves the optimality of any solution of Problem (2.7) for Problem (4.4), and equivalently, for Problem (2.10).

Conversely, if $I_{* *}$ is an optimal solution of Problem (4.4), then the above proof shows that necessarily

$$
\int_{0}^{\infty} f_{1}(x) I_{* *}^{\prime}(x) \mathrm{d} x=\int_{0}^{\infty} f_{1}(x) I_{*}^{\prime}(x) \mathrm{d} x,
$$

which means that $I_{* *}$ is also a solution of Problem (2.7).

\section{Remark 4.7.}

i. The fact that the two-constraint Problem (2.10) can be equivalently reduced to the one-constraint Problems (2.7) and (2.8) subject to (4.3), which are optimization problems from the perspective of only one party, may seem puzzling, given that Problem (2.10) is designed to factor in the joint interests of the insurer and reinsurer in the first place. Such anomaly can be heuristically explained in the light of the behavior of the functions $f_{1}$ and $f_{0}$, which satisfy $f_{1}(x)=-f_{0}(x)$ for all $x \notin\left[F_{X}^{-1}(\alpha \wedge \beta), F_{X}^{-1}(\alpha \vee \beta)\right.$ ) (see Remark 
4.4 (ii)). As $f_{1}$ and $f_{0}$ are always opposite in sign but the same in magnitude on the complement of $\left[F_{X}^{-1}(\alpha \wedge \beta), F_{X}^{-1}(\alpha \vee \beta)\right)$, ceding an additional unit of loss there leads to a decrease (resp. increase) in $f_{1}$ that is exactly offset by the increase (resp. decrease) in $f_{0}$. If $\lambda>1 / 2$, the decrease (resp. increase) in the objective function will outweigh the increase (resp. decrease), leading to an overall decrease (resp. increase) in the objective function. This explains why when $\lambda>1 / 2$, Problem (2.10) is solved by solely minimizing $\int_{0}^{\infty} f_{1}(x) I^{\prime}(x) \mathrm{d} x$ over $I \in \mathcal{I}$ subject to the constraints (4.3). Analogous explanations hold in the cases when $\lambda=1 / 2$ and $\lambda<1 / 2$.

ii. Further to (i), it should be emphasized that the reduction of the twoconstraint Problem (2.10) to the one-constraint Problems (2.7) and (2.8) heavily relies upon the character of $f_{1}$ and $f_{0}$ when both the insurer and reinsurer adopt VaR as their risk measures and should be perceived as the exception rather than the rule. This phenomenon is not expected to occur when other risk measures such as TVaR are prescribed, in which case the "exact offsetting" property of $f_{1}$ and $f_{0}$ described in (i) is lost and Problem (2.10) cannot be solved by the methods proposed in this article. In fact, the peculiarity observed in Proposition 4.6 is a manifestation of a further shortcoming of VaR when it is employed as a decision-making vehicle in risk management, in addition to many of its flaws documented, for example, in Basak and Shapiro (2001) and Dowd and Blake (2006).

iii. In addition to Problem (2.10), Cai et al. (2016) (see Problem (25) therein) also considered the insurer-reinsurer symbiotic risk minimization problem subject to the insurer's loss constraint and the reinsurer's profit constraint:

$$
\left\{\begin{array}{cc}
\inf _{I \in \mathcal{I}} & \lambda \operatorname{VaR}_{\alpha}\left(X-I(X)+P_{I(X)}\right)+(1-\lambda) \operatorname{VaR}_{\beta}\left(I(X)-P_{I(X)}\right) \\
\text { s.t. } & \operatorname{VaR}_{\alpha}\left(X-I(X)+P_{I(X)}\right) \leq \pi_{1} \\
& \mathbb{P}\left(P_{I(X)}-I(X) \geq L_{2}\right) \geq \gamma
\end{array},\right.
$$

where $L_{2}$ is the profit target and $\gamma$ is the confidence level of the reinsurer. Although the reinsurer's profit constraint is equivalent to $\operatorname{VaR}_{\gamma}[I(X)-$ $\left.P_{I(X)}\right] \leq-L_{2}$, whose structure is identical to the reinsurer's risk constraint in Problem (2.10), note that the two confidence levels $\beta$ and $\gamma$ need not be the same, in which case three functions, $f_{0}, f_{1}$ defined in (4.1), and $f_{2}$ defined by $f_{2}(x):=1_{\left\{S_{X}(x)>1-\gamma\right\}}-(1+\theta) S_{X}(x)$ need to be dealt with. Therefore, unless $\beta=\gamma$, Problem (4.5) is essentially different from Problem (2.10) and requires further analysis.

\section{CONCLUDING REMARKS}

Constrained optimal reinsurance is a mathematically challenging and practically significant research problem that has constantly attracted a great deal of interest from researchers and practitioners. This article addresses constrained 
optimal reinsurance problems by drawing upon their intrinsic Neyman-Pearson nature, which puts these problems into perspective and allows for a unifying, transparent and thorough treatment. The Neyman-Pearson-type explicit solutions obtained by specializing the general solution scheme to three concrete constrained optimal reinsurance problems demonstrate that many of them were only partially and unnecessarily tediously analyzed by ad hoc methods in the literature, but are now completely and methodically solved. It is anticipated that the usefulness of such a Neyman-Pearson approach will extend beyond the realm of optimal reinsurance to many other constrained optimization problems in risk management and insurance which are partly or not at all statistical in character.

We remark that while there are generalizations of the classical NeymanPearson Lemma to the case of multiple inequality constraints in the mathematics and statistics literature (see, for example, Property 3.5 of Francis and Wright, 1969, Proposition 6.1 of Shao, 2003, and Theorem 3.6.1 of Lehmann and Romano, 2005), they merely provide sufficient conditions for the constrained optimization problem to admit optimal solutions. These sufficient conditions are hard to ascertain in practice, and that clear-cut characterizations given in Theorem 3.2 of this article are hardly available. The solution of optimal reinsurance problems in the presence of several external constraints remains an insurmountable problem that requires future research.

\section{ACKNOWLEDGEMENTS}

The author would like to thank the anonymous reviewers and Haibo Liu at the University of Iowa for their careful reading and insightful comments. Support from a start-up fund provided by the College of Liberal Arts and Sciences, The University of Iowa, and a Centers of Actuarial Excellence (CAE) Research Grant (2013-2016) from the Society of Actuaries is gratefully acknowledged.

\section{NOTES}

1. Suppose by way of contradiction that $g\left(0_{+}\right)>0$. For any unbounded non-negative random variable $Y$, whose survival function satisfies $S_{Y}(y)>0$ for all $y \geq 0$, the non-decreasing monotonicity of $g$ leads to $\rho_{g}(Y)=\int_{0}^{\infty} g\left(S_{Y}(y)\right) \mathrm{d} y \geq g\left(0_{+}\right) \int_{0}^{\infty} \mathrm{d} y=\infty$.

2. To be precise, every $I \in \mathcal{I}$ should be defined on $\left[0, F_{X}^{-1}(1)\right)$ rather than $\mathbb{R}^{+}$and every integral $\int_{0}^{\infty}$ in this article should mean $\int_{0}^{F_{X}^{-1}(1)}$, but we choose not to overload notations.

3. Strictly speaking, Problem (2.10) is not directly covered by Problem (3.1) because of the presence of two constraints. However, it is shown in Subsection 4.3 that Problem (2.10) can be converted into an equivalent one-constraint problem covered by Problem (3.1).

4. Our analysis remains applicable if one replaces the integrals $\int_{0}^{\infty} f_{1}(x) I^{\prime}(x) \mathrm{d} x$ and $\int_{0}^{\infty} f_{0}(x) I^{\prime}(x) \mathrm{d} x$ in Problem (3.1) by $\int_{0}^{\infty} f_{1}(x) I^{\prime}(x) \mathrm{d} \mu(x)$ and $\int_{0}^{\infty} f_{0}(x) I^{\prime}(x) \mathrm{d} \mu(x)$, respectively, for any measure $\mu$ on the non-negative real line, but there is no need for such additional generality in this paper. 


\section{REFERENCES}

ARrow, K. (1963) Uncertainty and the welfare economics of medical care. American Economic Review, 53, 941-973.

Balbás, A., BALbÁs, B., BAlbás, R. and Heras, A. (2015) Optimal reinsurance under risk and uncertainty. Insurance: Mathematics and Economics, 60, 61-74.

BASAK, S. and SHAPIRO, A. (2001) Value-at-risk-based risk management: Optimal policies and asset prices. Review of Financial Studies, 14, 371-405.

Belles-SAmpera, J., Guillen, M. and SANTOlino, M. (2016) What attitudes to risk underlie distortion risk measure choices? Insurance: Mathematics and Economics, 68, 101-109.

BorCH, K. (1960) An attempt to determine the optimum amount of stop loss reinsurance. Transactions of the 16th International Congress of Actuaries, 1, 597-610.

CAI, J., FANG, Y., LI, Z. and WiLLMOT, G.E. (2013) Optimal reciprocal reinsurance treaties under the joint survival probability and the joint profitable probability. The Journal of Risk and Insurance, 80, 145-168.

CAI, J., LemieuX, C. and LiU, F. (2016) Optimal reinsurance from the perspectives of both an insurer and a reinsurer. ASTIN Bulletin, 46, 815-849.

CAI, J. and TAN, K.S. (2007) Optimal retention for a stop-loss reinsurance under the VaR and CTE risk measure. ASTIN Bulletin, 37, 93-112.

CAI, J., TAN, K.S., WENG, C. and ZHANG, Y. (2008) Optimal reinsurance under VaR and CTE risk measures. Insurance: Mathematics and Economics, 43, 185-196.

ChEUNG, K.C. (2010) Optimal reinsurance revisited - a geometric approach. ASTIN Bulletin, 40, 221-239.

Cheung, K.C., LiU, F. and YAM, S.C.P. (2012) Average Value-at-Risk minimizing reinsurance under Wang's premium principle with constraints. ASTIN Bulletin, 42, 575-600.

CHEUNG, K.C. and LO, A. (2017) Characterizations of optimal reinsurance treaties: A cost-benefit approach. Scandinavian Actuarial Journal, 2017, 1-28.

CHI, Y. and TAN, K.S. (2011) Optimal reinsurance under VaR and CVaR risk measures: A simplified approach. ASTIN Bulletin, 41, 487-509.

CUI, W., YANG, J. and WU, L. (2013) Optimal reinsurance minimizing the distortion risk measure under general reinsurance premium principles. Insurance: Mathematics and Economics, 53, $74-85$.

Cummins, J.D. and MAHUL, O. (2004) The demand for insurance with an upper limit on coverage. The Journal of Risk and Insurance, 71, 253-264.

Dhaene, J., VAnduffel, S., Goovaerts, M.J., KAas, R., TANG, Q. and Vyncke, D. (2006) Risk measures and comonotonicity: A review. Stochastic Models, 22, 573-606.

DowD, K. and BlAKE, D. (2006) After VaR: The theory, estimation and insurance applications of quantile-based risk measures. The Journal of Risk and Insurance, 73, 193-229.

FRANCIS, R.L. and WRIGHT, G.P. (1969) Some duality relationships for the generalized neymanpearson problem. Journal of Optimization Theory and Applications, 4, 394-412.

Lehmann, E.L. and Romano, J.P. (2005) Testing Statistical Hypotheses, 3rd edition. New York: Springer.

Lo, A. (2016) A unifying approach to risk-measure-based optimal reinsurance problems with practical constraints. Scandinavian Actuarial Journal (in press), DOI: 10.1080/ 03461238.2016 .1193558$.

Lu, Z., Meng, L., WANG, Y. and Shen, Q. (2016) Optimal reinsurance under VaR and TVaR risk measures in the presence of reinsurer's risk limit. Insurance: Mathematics and Economics, 68, 92-100.

RAO, M.M. (2014) Stochastic Processes - Inference Theory, 2nd edition. New York: Springer.

SHAO, J. (2003) Mathematical Statistics, 2nd edition. New York: Springer.

TAN, K.S., WENG, C. and ZHANG, Y. (2011) Optimality of general reinsurance contracts under CTE risk measure. Insurance: Mathematics and Economics, 49, 175-187.

WAGNER, D.H. (1969) Nonlinear functional versions of the Neyman-Pearson Lemma. SIAM Review, 11, 52-65.

ZHENG, Y. and CUI, W. (2014) Optimal reinsurance with premium constraint under distortion risk measures. Insurance: Mathematics and Economics, 59, 109-120. 
Zhuang, S.C., Weng, C., TAN, K.S. and Assa, H. (2016) Marginal Indemnification Function formulation for optimal reinsurance. Insurance: Mathematics and Economics, 67, 65-76.

AMBROSE Lo (Corresponding author)

Department of Statistics and Actuarial Science

The University of Iowa

241 Schaeffer Hall, Iowa City

IA 52242-1409, USA

Tel.: (319) 335-1915

E-Mail:ambrose-lo@uiowa.edu 